\title{
Planar Geometric Projections and Viewing Transformations
}

INGRID CARLBOM

Program in Computer Science, Brown University, Providence, Rhode Island 02912

JOSEPH PACIOREK

Computervision Corporation, Bedford, Massachusetts 01730

Illustrated by Thomas K. Stat, Rhode Island School of Design, Providence, Rhode Island

\begin{abstract}
In computer graphics one is often concerned with representing three-dimensional objects on a two-dimensional display surface. The choice of such a representation depends on several factors, including the purpose for which the representation is intended, the visual effects that are desired, and the shape of the object. This paper describes how twodimensional views can be obtained using planar geometric projections such as perspective and parallel projections. It discusses how these projections can be generated from a threedimensional representation of an object in a manner suitable for computer graphics systems. In particular, it shows how these projections can be generated using the viewing transformations of the Core Graphics System. The factors that affect the choice of projection are also discussed, and some guidelines for making such a choice are given.

Keywords and Phrases: computer graphics, viewing transformations, descriptive geometry, engineering drawing, architectural drawing, planar geometric projections, perspective projections, parallel projections.
\end{abstract}

CR Categories: $1.3,3.20,3.41,5.0,8.2$

\section{INTRODUCTION}

In computer graphics one is often concerned with representing three-dimensional objects on a two-dimensional display surface. Such a representation may attempt either to show the general appearance of an object, as in a photograph, or to depict the object so that its metric properties such as distances and angles can easily be derived. These methods of representation, as well as the representations themselves, are known as projections.

To produce a two-dimensional view of an object, each point of the object must be mapped onto a plane. The kind of mapping that is used distinguishes the types of projection and the resulting visual effects. This paper describes how two-dimensional projections can be generated from a three-di- mensional representation of an object, and discusses the visual advantages and disad. vantages of the various types of projections. This paper also illustrates how these projections can be generated with the viewing transformations in the Core Graphics System [GSPC77, BERG78].

The projections treated in this paper are known as planar geometric projections. A planar geometric projection of an object is obtained by passing lines called projectors, one through each point of the object, and finding the image formed by the intersections of these projectors with a plane of projection. The projectors emanate from a single point called the center of projection. When this point is finite, a perspective projection is obtained. When it is at infinity, that is, when the projectors are all parallel, a parallel projection is obtained. A perspec-

Permission to copy without fee all or part of this material is granted provided that the copies are not made or distributed for direct commercial advantage, the ACM copyright notice and the title of the publication and its date appear, and notice is given that copying is by permission of the Association for Computing Machinery. To copy otherwise, or to republish, requires a fee and/or specific permission.

(C) 1978 ACM 0010-4892/78/1200-0465 $\$ 00.75$ 
CONTENTS

INTRODUCTION

1. HISTORY OF PROJECTIONS

2. APPLICATIONS OF PROJECTIONS

2.1 Multiview Orthographic Projections

2.2 Axonometric Projections

2.3 Oblique Projections

2.4 Pexspective Projections

2.5 Summary

3. PLANAR GEOMETRIC PROJECTIONS

3.1 Multiview Orthographic Projections

3.2 Axonometric Projections

3.3 Oblique Projections

3.4 Perspective Projections

4. SPECIFICATIONS OF VIEWING TRANSFORMATIONS IN A GRAPHICS SYSTEM

4.1 Parallel Projections

4.2 Perspective Projections

CONCLUSION

ACKNOWLEDGMENTS

APPENDIX

A.1 Homogeneous Coordinates and Matrix Representations

A.2 Direction Cosines

A.3 Spherical Coordinates

A.4 Constraining Equations for Axonometric Projections

A.5 Conditions That Determine an Axonometric Projection

A. 6 Conditions That Determine an Oblique Projection

A.7 Conditions That Classify Perspective Projections

A.8 Projection Matrices

A.9 Viewing Specification in the Core Graphics System REFERENCES

tive projection illustrates the general appearance of an object as it would be seen by the eye, whereas a parallel projection primarily attempts to represent its metric properties. This paper discusses both perspective and parallel projections with their subclassifications. The Core Graphics System was designed to provide a set of viewing functions that can generate all planar geometric projections.

There are many types of projections that are beyond the scope of this paper. The projection of an object can be obtained by a method that does not use straight line projectors or that does not use a plane as a projection surface. By proper choice of projections, it is possible to preserve properties that are generally not preserved by planar geometric projections, such as shape, scale, and area. Non-planar and non-geometric projections are used extensively in cartography; for a discussion of these projections, the reader is referred to literature on map projections [KELL49, RICH72, STEE48].
The principles of planar geometric projections (hereafter referred to as projections) are found in descriptive geometry. This branch of geometry and its applications in engineering and architectural design are concerned both with drawing mathematically exact representations of objects, and with the properties of these two-dimensional representations. Projective geometry provides the theoretical basis for descriptive geometry and deals with those properties of objects that are invariant under projective transformations.

This paper discusses the methods of projection not in terms of two-dimensional drawing, but in terms of the position of the projection plane and the center of projection. It describes how these parameters determine the type of projection, and how they relate to the mathematical and visual properties of the two-dimensional representations.

For any given projection type, there are two approaches to obtaining a desired view of an object. One is to transform the object; the other is to choose new projection planes. The first approach is to use a fixed center of projection and projection plane, and to position the object to get the desired view. The second approach is to leave the object stationary and to choose the center of projection and projection plane so that the desired view is obtained. Although the first approach was long prevalent in descriptive geometry and its applications and has been used by most computer graphics systems, this paper uses the method of choosing projection planes. The methods are mathematically equivalent, but the method of choosing projection planes is better adapted to the approach taken by some computer graphics systems, including the synthetic camera approach of the Core Graphics System [NEwM78].

The choice of projection used to illustrate an object depends on a number of factors, including the purpose for which the representation is intended, the visual effects that are desired, and the shape of the object. This paper explains how these factors affect the choice of projection type and provides some guidelines for creating pleasing visual effects with each type of projection.

The first section below presents a short 


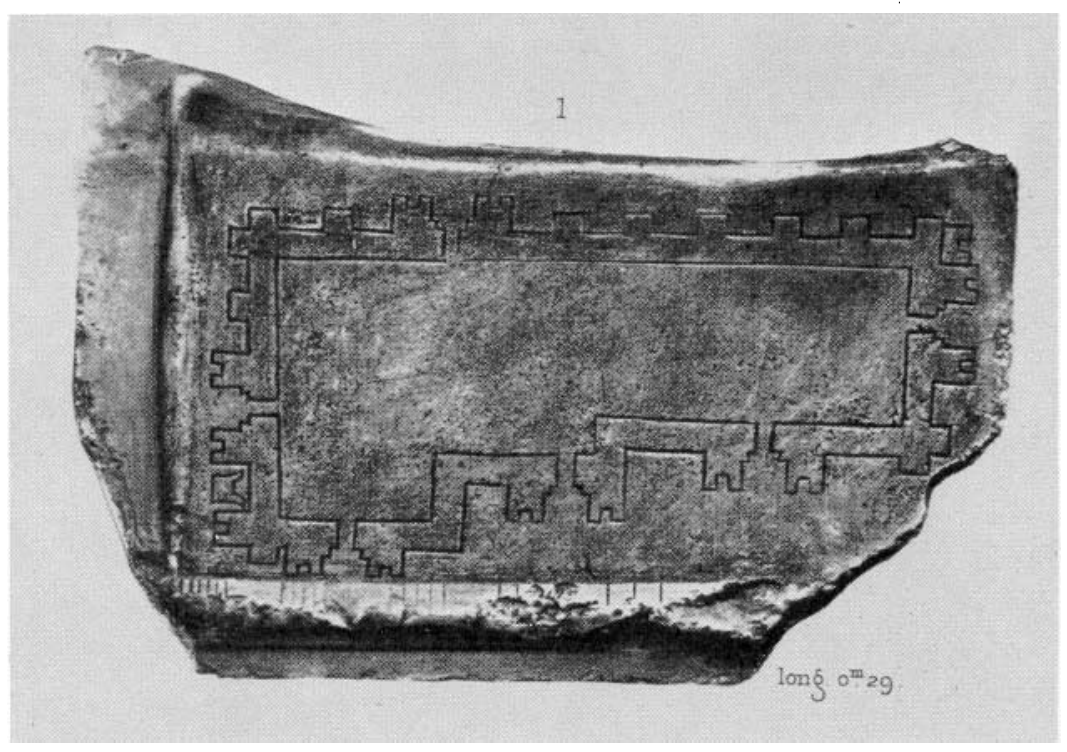

Figure 1-1. Plan view of building, part of statue of Gudea, from Lagash, Mesopotamia, c. 2150 B.C. (Ernest de Sarzec, Découvertes en Chaldée, 1891, Sterling Memorial Library, Yale University.)

history of the use of perspective and parallel projections in art, architecture, and engineering. The next section briefly introduces these projections and their subclassifications, and illustrates their use in current practice. In Section 3 the mathematical framework of the projections is developed, and their visual advantages and disadvantages are discussed. Section 4 introduces some viewing capabilities of the Core Graphics System and the use of these capabilities to specify the various types of projections. The Appendix contains mathematical derivations of the conditions that determine the different types of projections and some programming examples using the Core Graphics System, and illustrates a simple, straightforward way to implement the projections using homogeneous coordinate transformations.

\section{HISTORY OF PROJECTIONS ${ }^{1}$}

It is surmised that drawings have been used since early historic times to represent objects which were to be constructed. $\mathrm{Al}$ though no traces of these drawings are available today, it is not likely that early

\footnotetext{
${ }^{1}$ This section uses some terminology which is not
} defined until later sections. man could have built as accurately as he did without the use of fairly accurate drawings. The earliest known technical drawing in existence is that of a plan view of a building from about 2150 B.C. It is engraved on a stone tablet that is part of a statue representing Gudea, king of the city of Lagash in Mesopotamia: The engraving (Figure 1-1) is similar in form to the plan drawings used by architects today.

According to literary allusions, Greek painters and geometers during classical antiquity were acquainted with the laws of perspective. The painter Agatharchus (5th century B.C.) was the first to use perspective on a large scale. (Vases from as early as the late 6th century B.C. show isolated instances of its use.) Agatharchus wrote a book on "scene painting" which inspired the philosophers Anaxagoras and Democritus to write on perspective. During the 3rd century B.C., Euclid, Archimedes, and Apollonius studied the conic sections. Whereas Euclid and Archimedes investigated the metric properties of the conic sections, Apollonius studied those properties shared by all sections of a given cone. These properties, unlike the metric properties, are invariant under projective transformations. Apollonius' work provided the foundation for later work in projective geometry. 


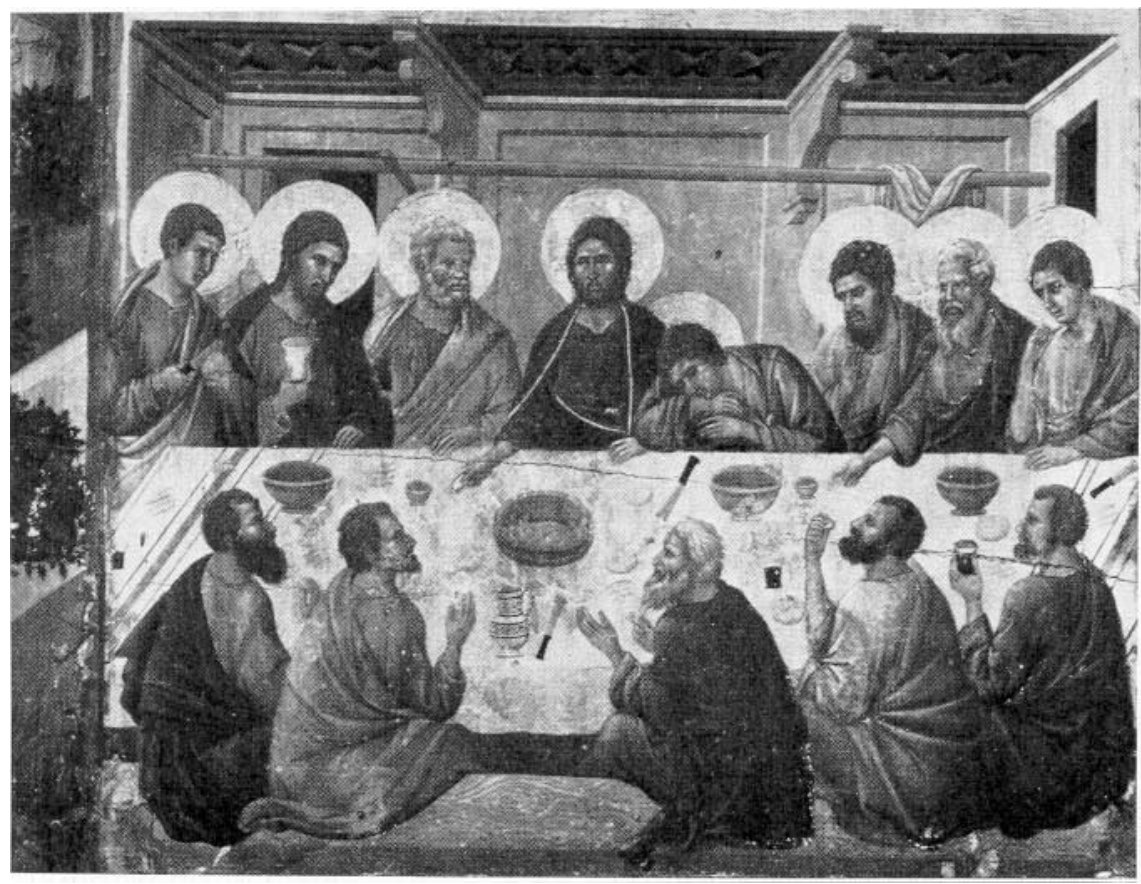

Figure 1-2. Duccio di Buoninsegna, The Last Supper, Opera del Duomo, Siena, fresco, c. 1308, (Alinari/ Editorial Photocolor Archives).

The first evidence of the use of drawings to guide construction work is found in the writings of Vitruvius, an architect and engineer in Rome under Julius Caesar and Augustus. About 14 B.C., he wrote $\mathrm{De}$ Architectura, which was intended as a complete guide for architects and engineers. Vitruvius discussed plan and elevation drawings of buildings and defined a perspective as a drawing where "the lines all meet at the center of a circle." Unfortunately, the illustrations that accompanied this work have been lost.

Although Greek artists had investigated the laws of perspective, these techniques were apparently not formalized until the beginning of the Renaissance. During medieval times, art was highly symbolic, generally illustrating religious events. Paintings from this time rarely give a sense of depth; people and objects seem two-dimensional. A green or brown line was used to indicate the ground, and objects farther away were depicted with horizontal or vertical displacements. About the beginning of the 14th century artists again began to take an interest in representing the real world in their works. Duccio (1255-1319) and Giotto (1276-1336) made efforts at illustrating the third dimension using perspective. In Duccio's The Last Supper (Figure 1-2), the receding walls and ceiling lines are foreshortened to suggest depth.

During the 15th century, artists, many of whom were also able mathematicians, realized that perspective could be explained in terms of geometry. The first artist to develop a mathematical system for perspective was Filippo Brunelleschi (13771446). The first treatise on perspective, Della Pittura, was published in 1435 by Leone Battista Alberti (1404-1472). Although Euclid, in Optica, had already described the system of vision as a cone or pyramid of visual rays emanating from the eye to an object, Alberti was the first to define a painting as a cross section of this visual pyramid. In his work, Alberti describes a method of drawing perspective, known as the focused system, by which an artist constructs a painting on a perspective grid. 
The techniques of perspective were further developed during the 15th century, most notably by Piero della Francesca (1420-1492) and Leonardo da Vinci (1452-1519). In his text, De Prospettiva Pingendi, Piero extended Alberti's work and described a method of constructing perspective from a top and a front view of an object. One of Piero's most famous paintings, The Resurrection (Figure 1-3), is an interesting study of perspective-it has been painted from two points of view, one opposite the center of the sarcophagus, the other opposite the face of Jesus Christ. The reader will notice that his attention is drawn alternately to the sarcophagus and to the face of Christ. ${ }^{2}$

One of Leonardo's most famous paint-

$\overline{2}$ This phenomenon is discussed further in Section 3.

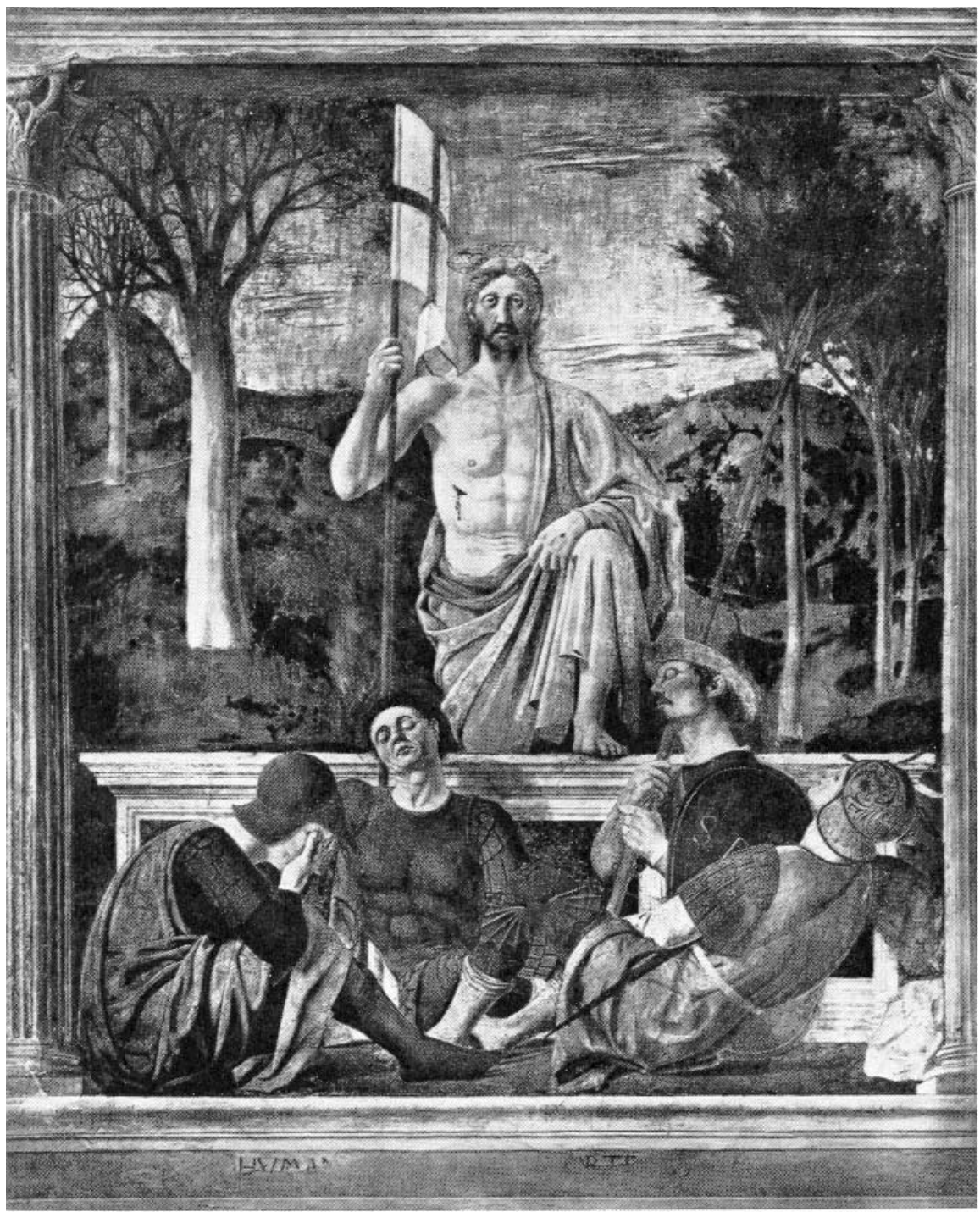

Figtre 1-3. Piero della Francesca, The Resurrection, Pinacoteca Civica, San Sepolero, fresco, c. 1460, (Alinari/Editorial Photocolor Archives). 


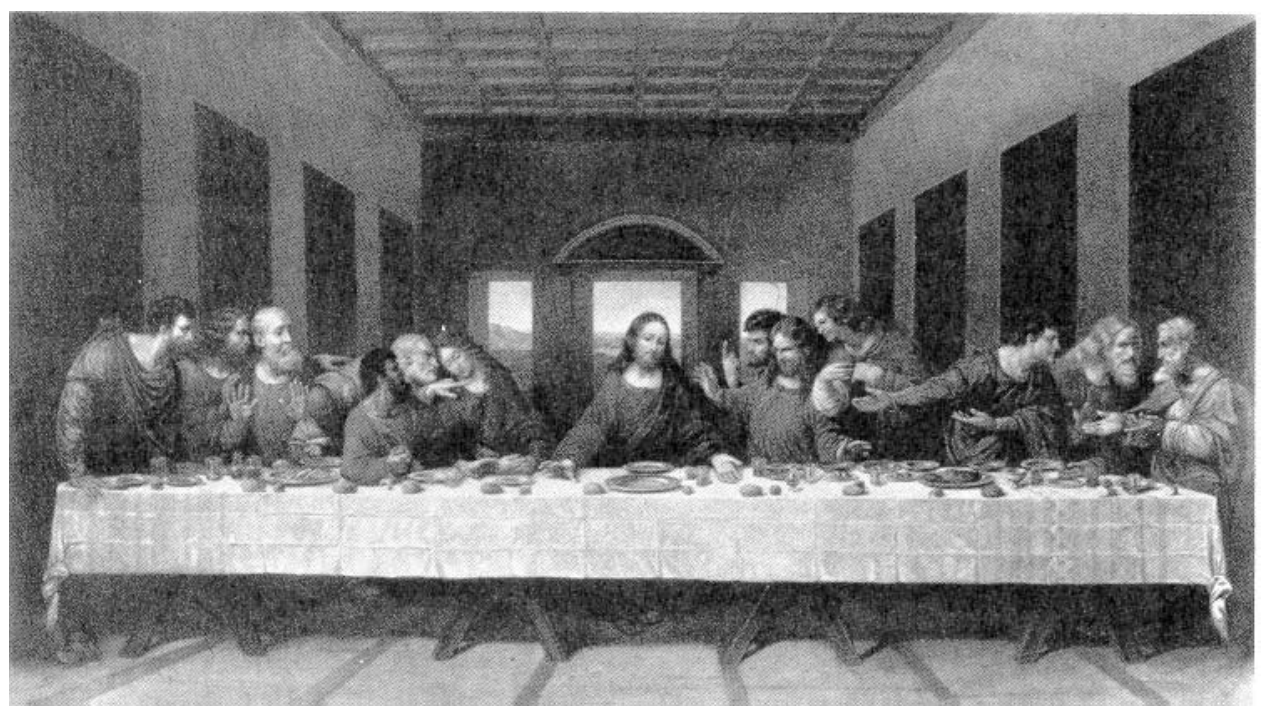

Figure 1-4. Engraving of Leonardo da Vinci's fresco, The Last Supper, Santa Maria delle Grazie, Milan, c. 1495-1498, (The Bettmann Archive).

ings, The Last Supper (Figure 1-4), is a perfect study of perspective. A comparison with Duccio's painting of the same name clearly demonstrates the development of the science of perspective during the 15th century. Although Duccio's work has some suggestion of depth, Leonardo gives the viewer a feeling of actually being in the room, watching a scene from real life.

The most widely read treatise on per- spective from this time was written not by one of the Italian masters, but by a German, Albrecht Dürer (1471-1528). In his work, Unterweysung der Messung mit dem Zyr. kel und Rychtscheyd, Dürer describes both mathematical and mechanical methods for drawing perspective. One of the mechanical methods for constructing a perspective view of an object is illustrated in his woodcut, Artist Drawing a Lute (Figure 1-5).

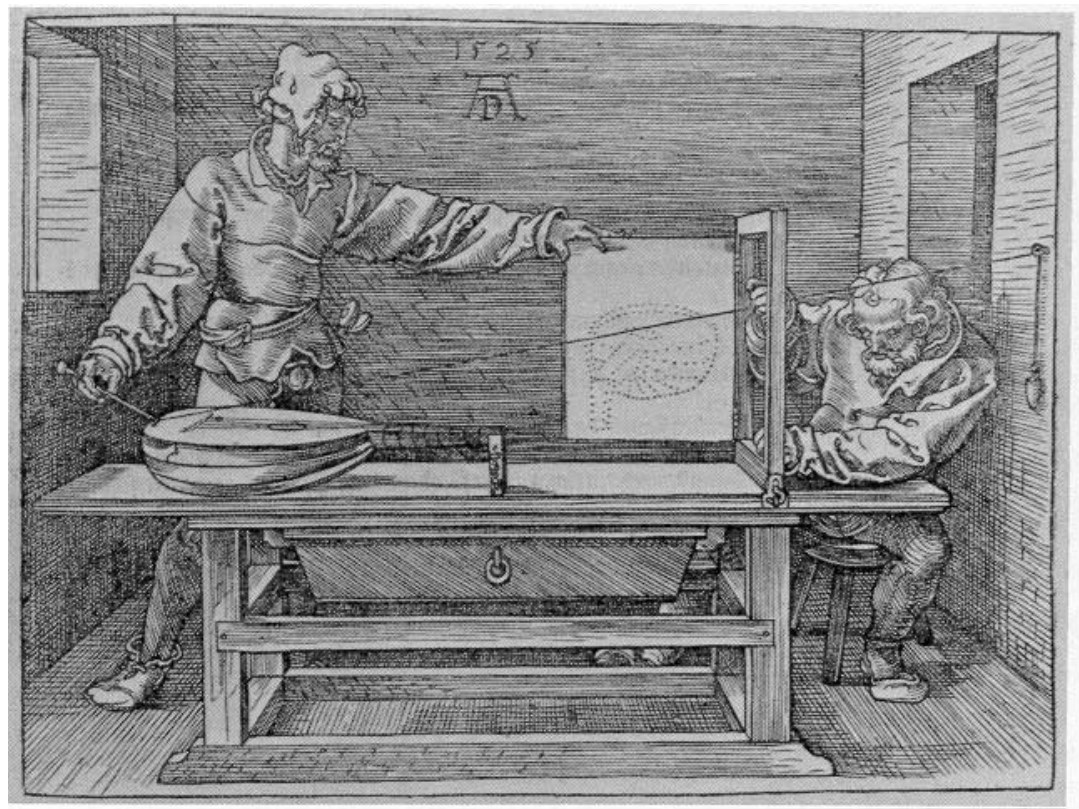

FIGURE 1-5. Albrecht Dürer, Artist Drawing a Lute, woodcut from Unterweysung der Messung mit dem Zyrkel und Rychtscheyd, 1525, (The Metropolitan Museum of Art, Harris Brisbane Dick Fund, 1941). 
Although it is believed that Dürer was familiar with the existing Renaissance texts on perspective, it seems he failed to understand the known methods of perspective drawing [IvIN75]. This is particularly evident in his copper engraving, St. Jerome in His Study (Figure 1-6). The choice of the principal vanishing point to the far right and the "wide angle" effect caused by choosing the station point very close to the room give a noticeably distorted view. ${ }^{3}$

The theory of perspective was further investigated by the French architect, engi-

\footnotetext{
$\overline{3}$ These types of distortion are discussed further in Section 3.
}

neer, and mathematician Gerard Desargues (1593-1662). In 1639, Desargues published a treatise on the conic sections, which laid the foundation for the study of projective geometry. His work was, however, ignored by his contemporaries, and all printed copies of his work were lost.

Multiview orthographic projections had been used during the Middle Ages by architects and during the Renaissance by artists as an aid in constructing perspective. The application of these projections to engineering drawing was first made in an organized fashion by Gaspard Monge (1746-1818), who is considered the "father of descriptive geometry." Monge developed

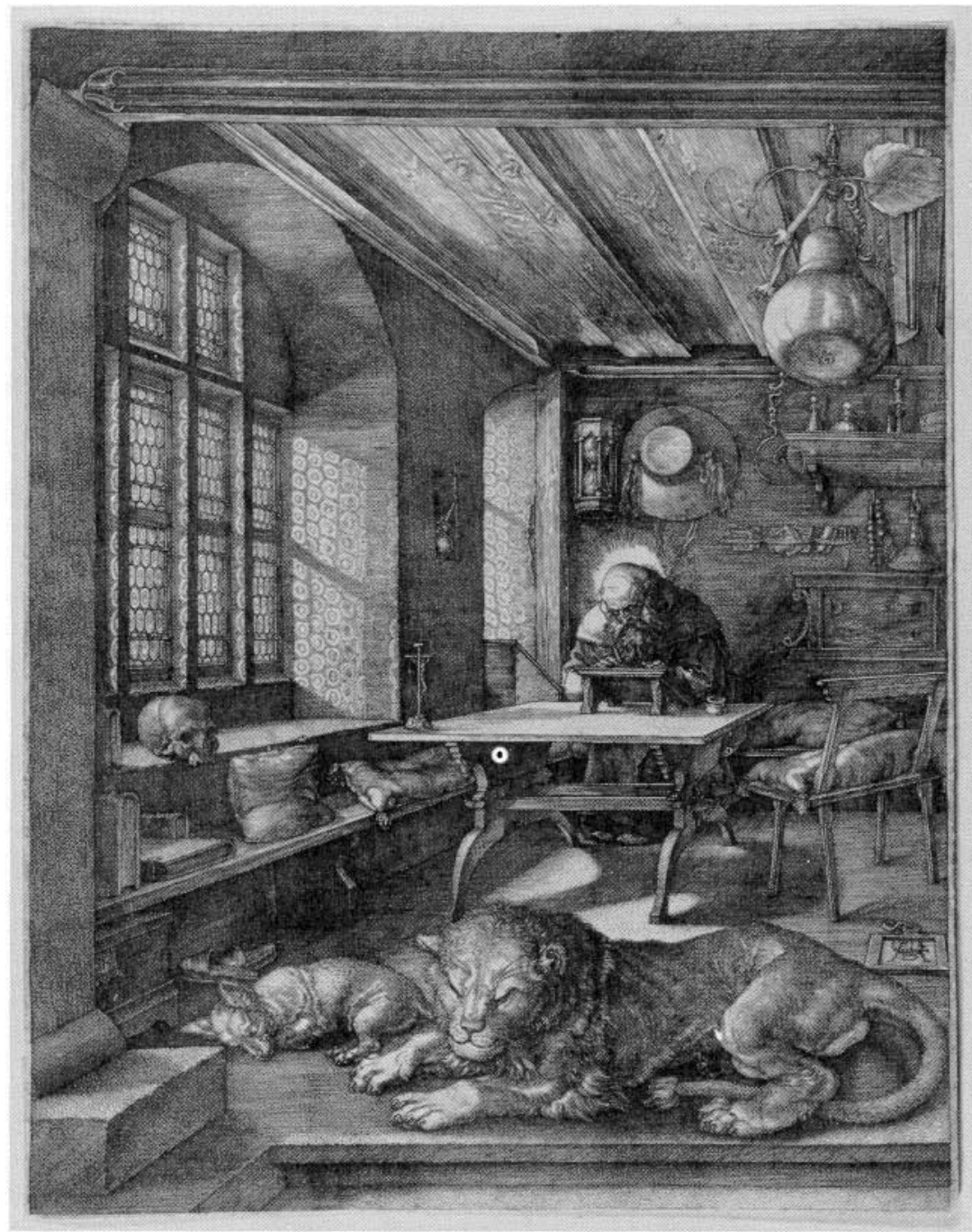

Figure 1-6. Albrecht Dürer, St. Jerome in His Study, engraving, 1514, (The Metropolitan Museum of Art, Fletcher Fund, 1919). 
his principles for the geometrical solution of spatial problems in 1765 while working as a draftsman of military fortifications. His solutions were at first regarded with disbelief, but were later guarded as a French military secret. Monge eventually became a professor of mathematics at l'Ecole Polytechnique and made his system of descriptive geometry public in his lecture notes, Leçons de Géometrie Descriptive, in 1795 and in his textbook, Géometrie Descriptive, first published in 1801 . One of Monge's students, Jean Victor Poncelet (1788-1867), revived the study of projective geometry, which became an individual branch of mathematics that attracted many able mathematicians during the 19 th century.

Monge's principles were introduced into the United States by another of his students, Claude Crozet, who began to teach descriptive geometry at West Point in 1816 . His treatise on the subject was published in 1821. In 1826, Crozet's successor at the military academy, Charles Davies, published the first extensive work in the US on descriptive geometry. In 1864, Albert Church, who had succeeded Davies, published his famous text, Elements of Descriptive Geometry, the leading textbook on the subject in this country through the first decade of the 20 th century. ${ }^{4}$

\section{APPLICATIONS OF PROJECTIONS}

This section briefly introduces the different types of projections according to their advantages and disadvantages for various applications. These applications range from layout drawings used in design, through working drawings for production, to presentation drawings of finished products. Drawings are used by architects to show the appearance of proposed buildings, by engineers to describe structures and machine parts, by designers to present proposed products, and by commercial artists to illustrate objects in catalogs and advertisements. This section discusses the visual effects of the projections; the following section describes the methods for generating

\footnotetext{
${ }^{4}$ The reader is referred to the bibliography for these references and for several 20th-century texts on descriptive geometry, engineering drawing, and architectural drawing.
}

the various types of projections and illustrates how these methods of projection determine the characteristics of the resulting two-dimensional representations.

The choice of a projection to represent a three-dimensional object in a flat drawing is determined by the purpose for which the representation is intended. This purpose is usually a compromise between the conflicting goals of illustrating the general appearance of an object, i.e., as it appears to the eye from some desired position, and that of clearly indicating the shape and measurements of the object. Another traditional consideration in choosing a projection is the ease with which a draftsman can construct the drawing. This is no longer a concern if the projection is generated by a computer system.

In general, the interpretation of the projection of an object depends on the training of the observer, on the type and amount of information about the object that is presented, and on the complexity of the object. For example, an orthographic projection shows the exact shape of one face of an object and is easy to draw. However, such a projection usually requires more than one view to represent the whole object and a great deal of experience is necessary to visualize its three-dimensional shape. Because this method of representation usually requires more than one view, it is referred to as a multiview orthographic projection. Perspective projection, on the other hand, provides the most realistic representation of an object and the observer can easily visualize the three-dimensional shape of the object. However, a perspective projection is the most difficult drawing to construct, and different parts of the object are represented at different scales. Axonometric and oblique projections combine the pictorial advantages of perspective with the advantage of illustrating some principal measurements to scale.

\subsection{Multiview Orthographic Projections}

A multiview' orthographic drawing shows the exact shape of two or more faces of an object. The number of views required to adequately describe the dimensions of an object depends on the complexity of its shape. A simple symmetrical object with 


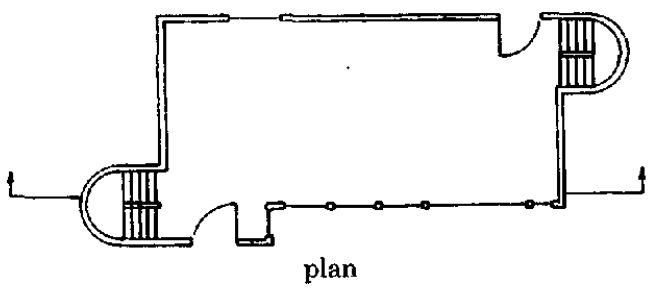

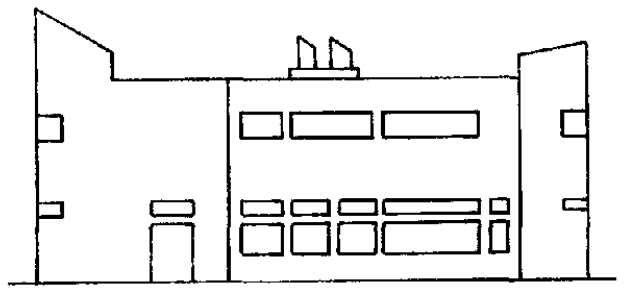

front elevation

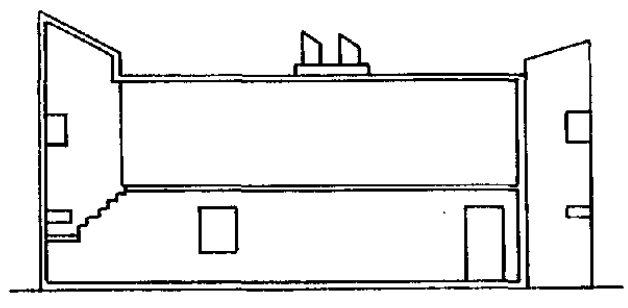

section

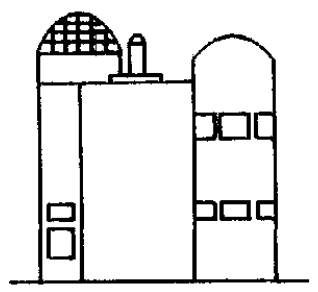

right elevation

FIgure 2-1. Multiview orthographic projection: plan, elevations, and section of a building.

rectangular faces can often be described in only two views. A more complicated object with inclined faces will need more. Objects with complicated internal detail may require one or more sectional views. Figure 2-1 illustrates a plan, a section, and a front and side elevation of a building.

Multiview orthographic projections are presented with the hidden lines either entirely omitted, or indicated as dashed lines. If the hidden lines are included, the information content of each view is increased, and fewer views are required. In Figure 2-1 the hidden lines are omitted, whereas in Figure 3-2a they are included.

Multiview orthographic projections are used for engineering drawings of machines and machine parts and for architectural working drawings of buildings.

\subsection{Axonometric Projections}

An axonometric projection usually repre- sents an object so that three adjacent faces are visible, in order to get a three-dimensional representation in one view. Such projections are particularly well suited to illustrate objects composed mostly of rectangular shapes (see Figure 2-2).

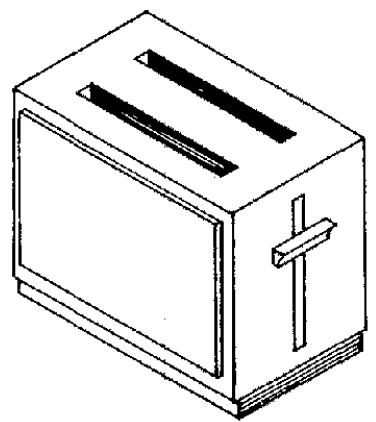

Figure 2-2. Axonometric projection is well suited for objects with mostly rectangular shapes. 
Although axonometric projections illustrate the three-dimensional shape of an object, axonometric views often seem distorted. This happens because more distant parts of an object are represented at the same scale as are close ones and the true shape of an object is rarely shown in these projections. For example, right angles are generally not represented as right angles, and circles are usually represented as ellipses. This is illustrated in Figure 2-3.

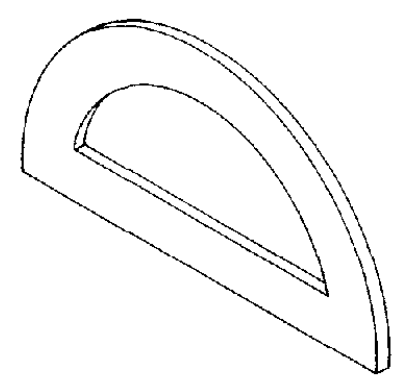

Figure 2-3. Axonometric projection. Circles usually appear as ellipses.

Axonometric projections are used in catalog illustrations, Patent Office records, piping diagrams, furniture design, machine design, and structural design.

\subsection{Oblique Projections}

An oblique projection has almost the same range of applications as an axonometric representation. However, while axonometric projections are primarily used for rectangular objects, oblique projections are also well suited for objects with cylindrical shapes. Oblique projections combine properties of orthographic projections with those of axonometric projections. An oblique projection provides the exact shape of one face of an object, and illustrates two adjacent faces in order to give a threedimensional representation in one view. Thus, an oblique projection is particularly suited for objects with much detail or irregular shapes on one principal face, as is illustrated in Figure 2-4.

Oblique projections produce distortions similar to those of axonometric projections. For example, receding parallel lines of the object are parallel in the drawing, but give the observer the illusion of divergence. This is illustrated in Figure 2-5.

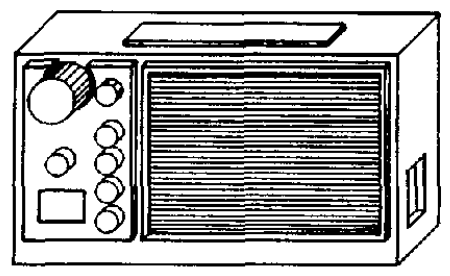

FIguRE 2-4. Oblique projection is well suited for objects with detail on one face.

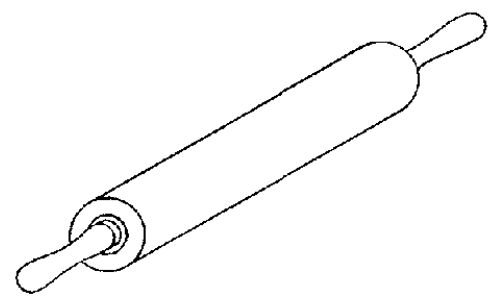

Figure 2-5. Oblique projection. Receding parallel lines give the illusion of divergence.

One common application of oblique projection is the so-called plan oblique drawing, which is used to illustr ite interior and exterior layouts of a building complex. Plan oblique drawings are also used in mapmaking, as is illustrated in Figure 2-6.

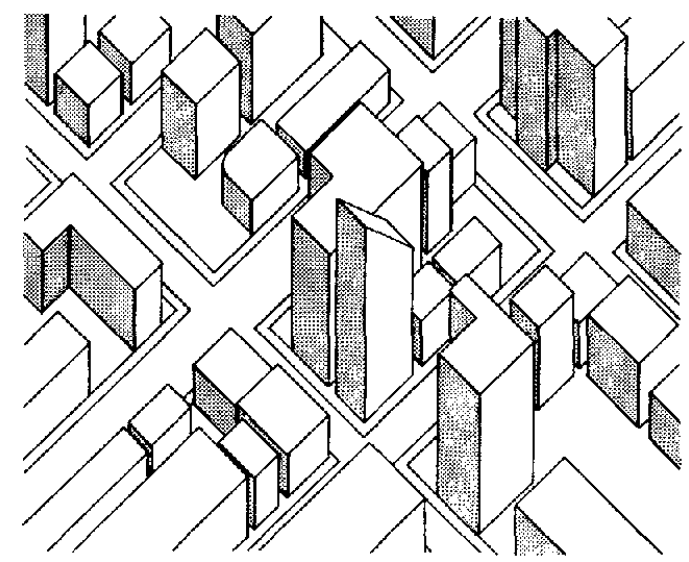

Figure 2-6. Plan oblique projection of a city. 


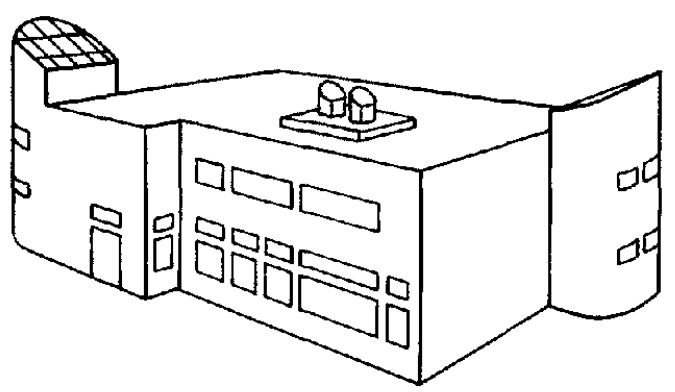

Figure 2-7. Perspective projection of a building.

\subsection{Perspective Projections}

A perspective projection represents an object as it would be seen by an observer positioned at a certain vantage point. An object appears smaller as its distance from the observer increases, and parallel lines of an object converge in the drawing. A perspective view of the building in Figure 2-1 is illustrated in Figure 2-7.

Perspective projections are not suitable for working drawings because it is difficult to determine the exact shape and size of an object from a perspective view. These projections are, however, widely used whenever a realistic appearance of an object is desired, such as in advertising and for presentation drawings in architectural, industrial, and engineering design.

\subsection{Summary}

Several different visual effects can be achieved with the projections discussed in this section. In practice, two or more types of projections are often used to complement each other so that several pictorial effects can be combined in one presentation. One example would be a merchandising catalog, which may use both a multiview orthographic projection and an axonometric projection to illustrate a machine part. Another example would be an architectural blueprint that may use a plan, a plan oblique, and a perspective view to describe a building.

\section{PLANAR GEOMETRIC PROJECTIONS}

This section describes how each type of planar geometric projection is obtained by the proper choice of a projection plane and a center of projection. The mathematical properties of each method of projection are discussed and related to the properties of the projected object. The derivations of these relationships can be found in the Appendix; only the results are described in this section.

Each type of projection provides a variety of visual effects. The previous section briefly discussed each type from the point of view of its applicability. This section further elaborates on the tradeoffs between the different types of projections. It also provides some suggestions for obtaining the most pleasing views of an object with each type of projection.

A projection, in this paper, denotes both a mapping of a three-dimensional space

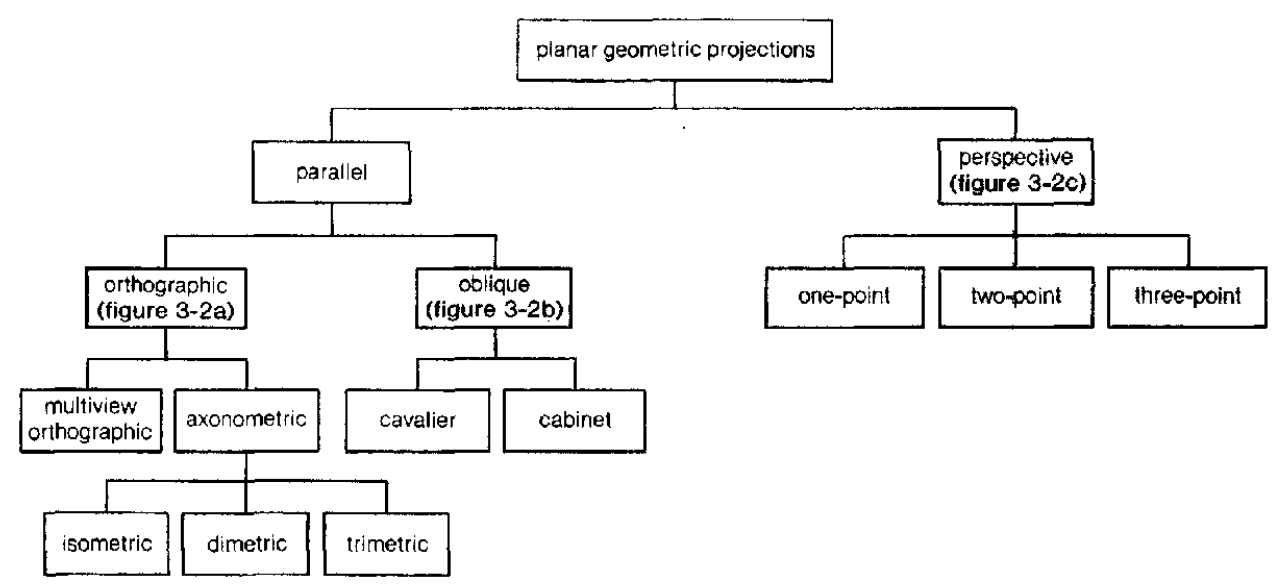

FIGURE 3-1. Classification of projections. 

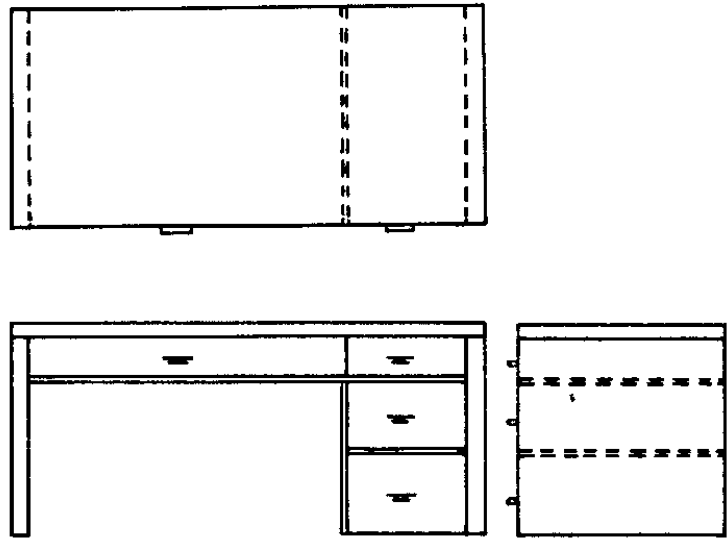

multiview orthographic
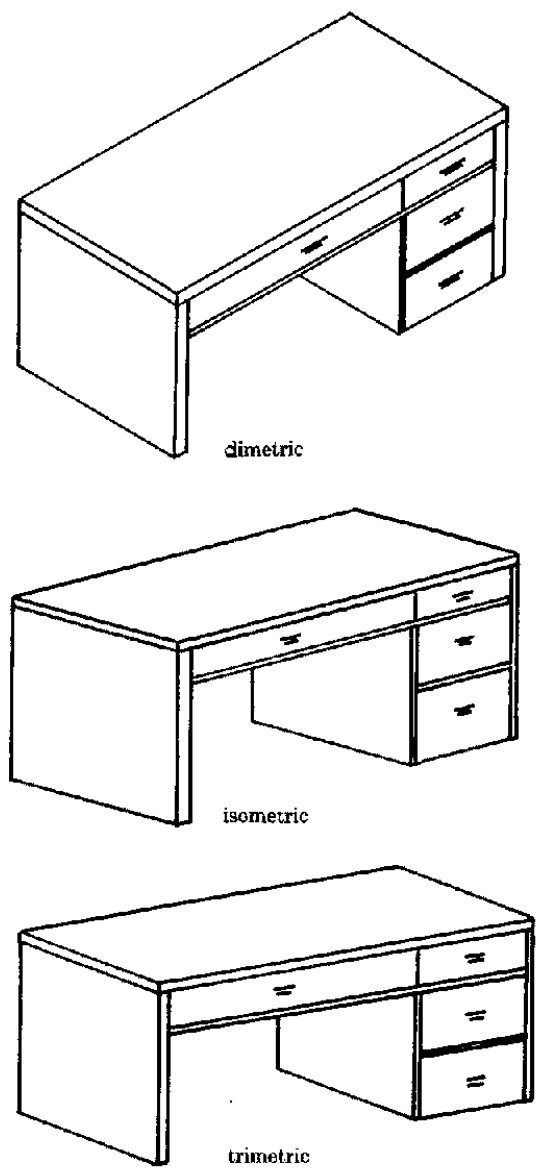

Figure 3-2a. Pictorial effects of orthographic projections.

onto a two-dimensional subspace, called the projection plane, and the resulting image of applying such a mapping to an object. The planar image of an object in three- dimensional space is found by passing a line through each point of the object and finding the intersections of these lines with the projection plane. These lines, the projectors, emanate from a single point called the center of projection. When the center of projection is at infinity, so that the projectors are all parallel, the projection is known as a parallel projection. When the center of projection is at a finite distance from the projection plane, a perspective projection results. Each of these two types has further subclassifications, which are illustrated diagramatically in Figure 3-1 and pictorially in Figure 3-2.

The classification of parallel projections is determined by the angle between the projectors and the projection plane. When the projectors are perpendicular to the projection plane, the projection is orthographic; otherwise, it is oblique. Orthographic projections are represented either as multiview orthographic projections or axonometric projections.

\section{A multiview orthographic projection is not a projec- tion as defined above but is a collection of such pro- jections. However, multiview orthographic projections are treated as a class of projections in this paper in accordance with common practice. \\ ${ }^{6}$ The hidden lines are indicated in the multiview or- thographic view. In all other illustrations in this sec- tion the hidden lines are omitted for presentational purposes.}

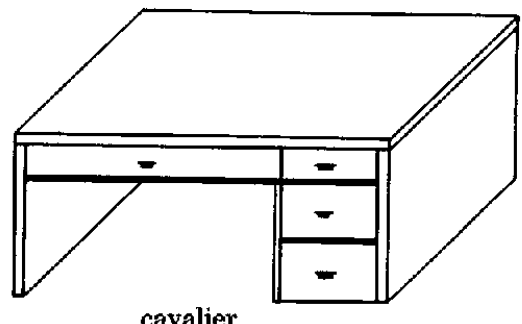

cavalier

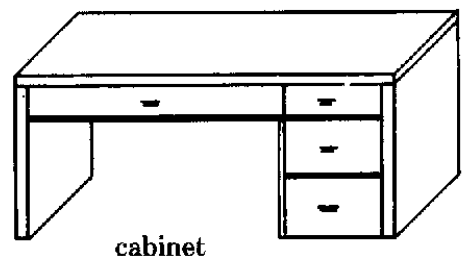

Figure 3-2b. Pictorial effects of oblique projections. 


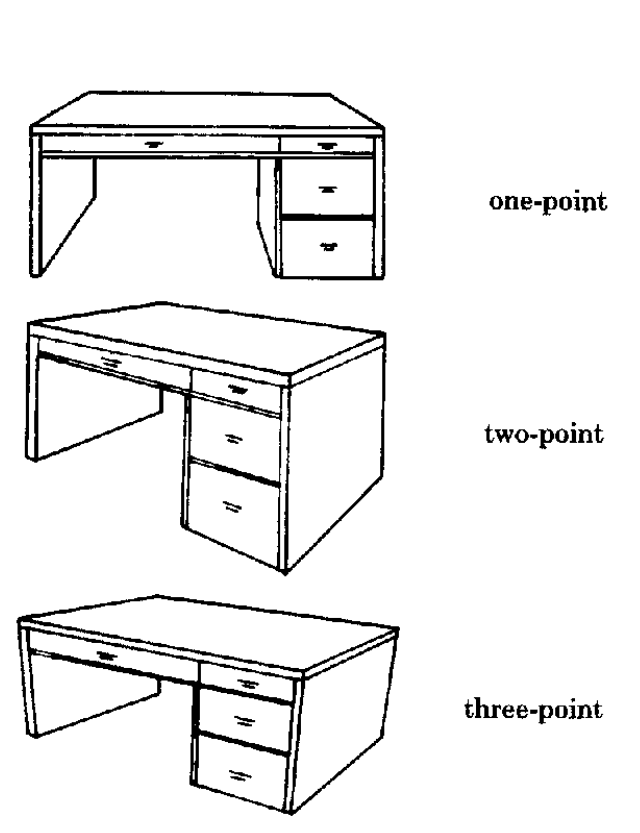

Figure 3-2c. Pictorial effects of perspective projections.

Most objects can be thought of as having three principal perpendicular axes. ${ }^{7}$ For convenience, the coordinate system is chosen to coincide with the principal directions of such an object. In the following discussion it is assumed that the coordinate system is chosen in this manner. Furthermore, in accordance with common practice, the

\footnotetext{
'For other objects, many distinctions between the
} projections discussed in this paper are no longer valid.

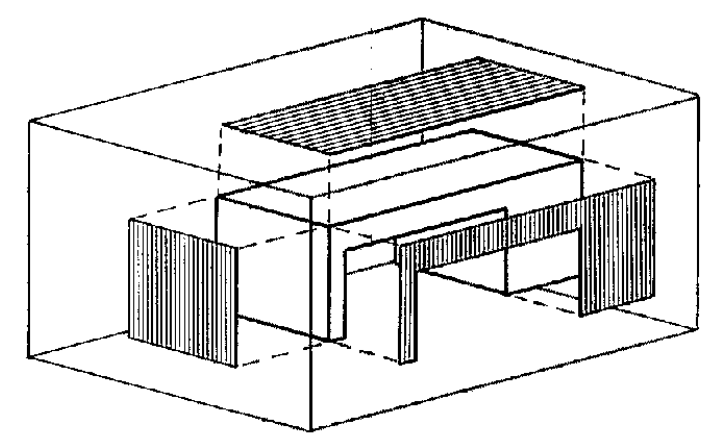

FIgure 3-3. Projecting orthographically onto three of the six prineipal planes.

two-dimensional images of the objects are positioned on the page with one principal axis as a vertical line.

\subsection{Multiview Orthographic Projections}

Multiview orthographic projections show, in one picture, two or more orthographic projections onto planes parallel to the principal planes. These projections are arranged relative to each other in a specified manner. A way of creating a multiview orthographic projection is to surround the object with six projection planes which form a rectangular box around the object, as shown in Figure 3-3. The six orthographic projections are then "unfolded" and arranged as illustrated in Figure 3-4.

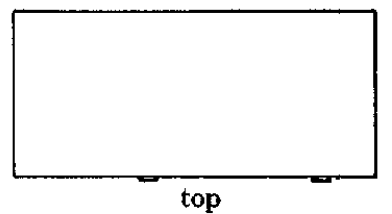

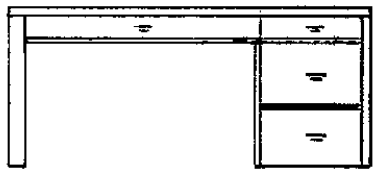

front

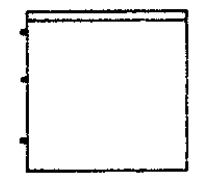

right side

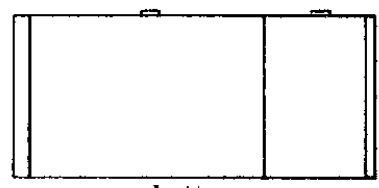

bottom

Figure 3-4. Multiview orthographic projection. The six principal orthographic views. 
Obviously, other arrangements of the six projections are possible, but this one is the most commonly used. Of these six projections, the top view is sometimes referred to as a plan, and the front and side views as front and side elevations.

Six views are rarely needed. For example, a simple, symmetrical object may be completely described by two or three views. The most common combination is top, front, and right side view. By convention each projection occupies a standard position relative to the others, no matter how many are used.

The principal multiview orthographic projections are well suited to describe the shapes of objects that are essentially rectangular. However, auxiliary views are required to describe the true shape of an object with faces inclined to the principal planes. Auxiliary views are orthographic projections onto planes inclined to the principal planes and parallel to the faces of

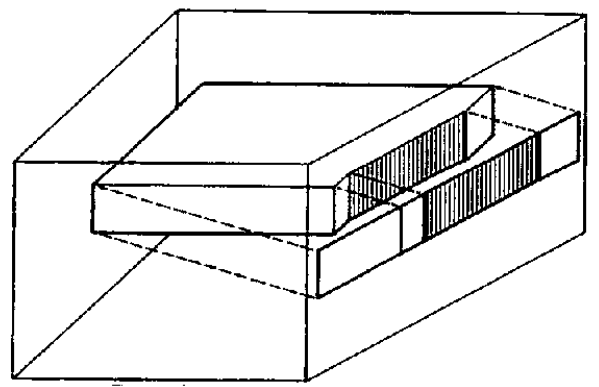

Figure 3-5a. Projecting orthographically onto an auxiliary plane.

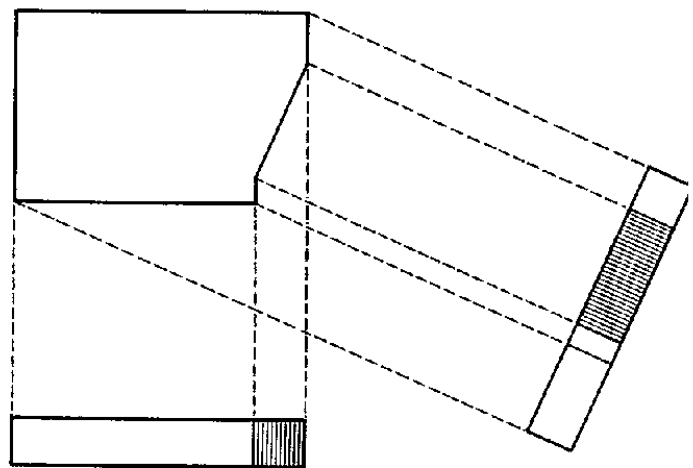

Figure 3-5b. Multiview orthographic projection consisting of two principal orthographic views and one auxiliary view. (Dashed lines indicate relationships of the three views.) interest. An auxiliary plane and the resulting orthographic projection is illustrated in Figure 3-5.

All the multiview projections discussed so far illustrate the exterior of an object. To represent objects with complicated interior detail, sectional views are used. A sectional view of an object is obtained by "cutting" the object with a plane, removing one part of the object, and projecting the remaining part orthographically onto the cutting plane. When the cutting plane is horizontal, the sectional view is generally referred to as a plan; when it is vertical, the sectional view is called simply a section. A plan and a section are illustrated in Figure 2-1. (The arrows in the plan indicate the section line, i.e., the position of the cutting plane.)

\subsection{Axonometric Projections}

An axonometric projection is an orthographic projection onto a single plane, where this plane is chosen in such a way that the general three-dimensional shape of an object is illustrated. It usually represents an object so that three adjacent faces are visible, but the true shape and size of any of these faces are not shown unless the face is parallel to the projection plane. In an axonometric projection, parallel lines are equally foreshortened. In particular, axonometric projections produce uniform foreshortening along the projected principal axes; thus, measurements can easily be made to scale along these axes.

The axonometric projections are classified according to the orientation of the projection plane, i.e., the angles between the projection plane and the coordinate axes. If all three angles are equal, the projection is isometric; if only two angles are equal, the result is a dimetric projection. If all angles are different, the projection is trimetric.

The type of axonometric projection determines the properties of the projected object, namely:

1) the number of foreshortening ratios ${ }^{8}$ of the principal coordinate axes that are equal, or

2) the number of angles between the projected coordinate axes that are equal.

\footnotetext{
${ }^{8}$ The foreshortening ratio of a line is its projected length divided by its true length.
} 


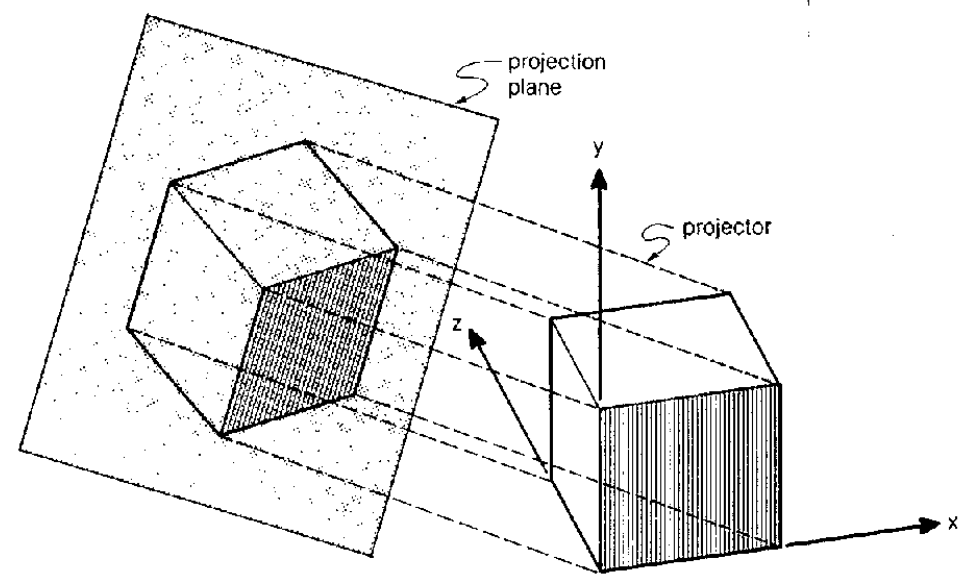

Figure 3-6a. Construction of an isometric projection.

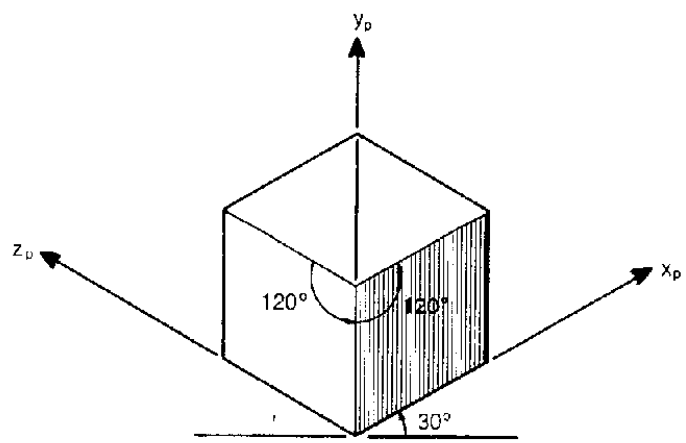

FIGURE 3-6b. Isometric projection resulting from the construction in Figure 3-6a. $\left(x_{p}, y_{p}\right.$ and $z_{p}$ are the projected coordinate axes.)

The mathematical equivalences between the orientation of the projection plane and these properties are shown in Section A.5 of the Appendix.

In an isometric projection all three coordinate axes are equally foreshortened, and the angles between the projected axes are all equal. To obtain an isometric projection, the projection plane must intersect all three coordinate axes at the same angle. As is shown in Section A.4 of the Appendix, this means that the projection plane normal must be parallel to one of the four lines $\pm x= \pm y= \pm z$. Hence there are only eight possible isometric views of an object. A cube, along with an isometric projection plane, the projectors, and the projected cube, is illustrated in Figure $3-6 .^{9}$

In a dimetric projection only two coordinate axes are equally foreshortened, and

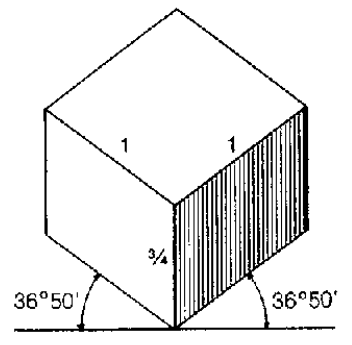

scale ratios $1: 3 / 4$

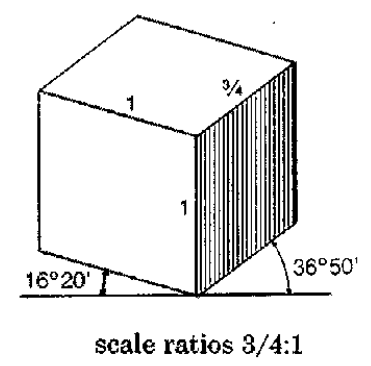

Figure 3-7. Dimetric projections of a cube.

only two of the angles between the projected axes are equal. Two different dimetric views of a cube are illustrated in Figure 3-7. ${ }^{10}$ To obtain a dimetric projection, the projection plane must intersect two of the coordinate axes at the same angle. As is shown in Section A.4 of the Appendix, this means that the projection plane normal must be parallel to one of the six planes $x= \pm y, x= \pm z$, or $y= \pm z$. By moving the projection plane normal in one of these planes, the emphasis of the three perpendicular faces of an object can be varied.

\footnotetext{
${ }^{9}$ Figure $3-6$ a shows how an isometric projection is constructed. This figure is itself a trimetric projection. The same is true for later figures which illustrate the construction of oblique and perspective projections. ${ }^{30}$ The scale ratios are displayed in the figure rather than the foreshortening ratios. These ratios more clearly illustrate the relative sizes of the sides of the cubes. The scale ratios are derived by multiplying the foreshortening ratios by the same scalar.
} 


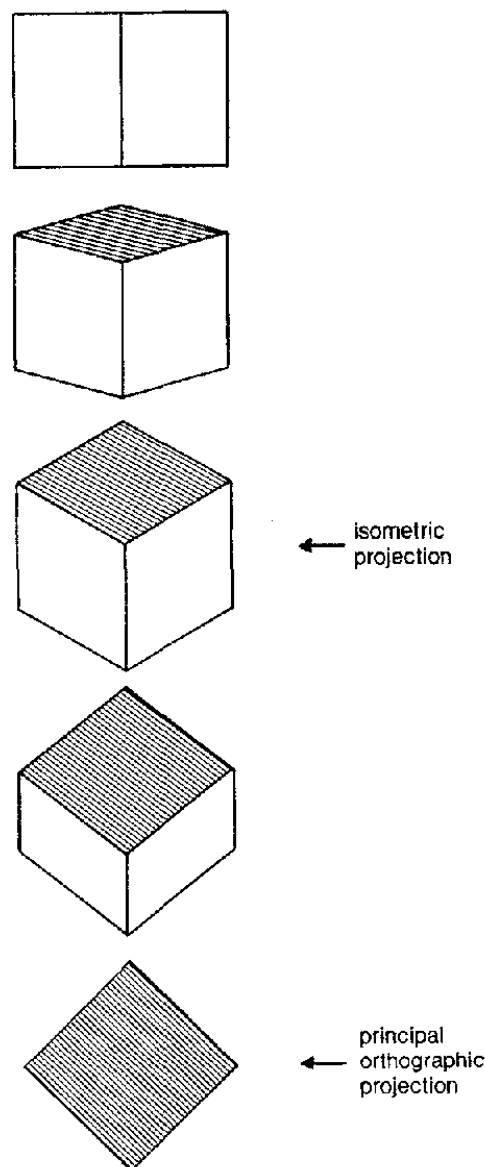

Frgure 3-8. Sequence of dimetric projections of a cube.

This is illustrated in Figure 3-8. (Note that the middle view is an isometric projection and that the last view is a principal orthographic view.)

A trimetric projection is the general form of axonometric projection. It produces different foreshortening of the three coordinate axes, and none of the angles between the projected axes are equal. Four different trimetric projections of a cube are shown in Figure 3-9.

The shape of an object determines the choice of a suitable axonometric representation. In order to minimize distortion and provide the most realistic view, the largest area, or the area with the most detail, should be emphasized. An isometric projection provides little freedom in choice of projection plane, and equal importance is given to all three principal faces, as is illustrated by the bookcase in Figure 3-10a. The
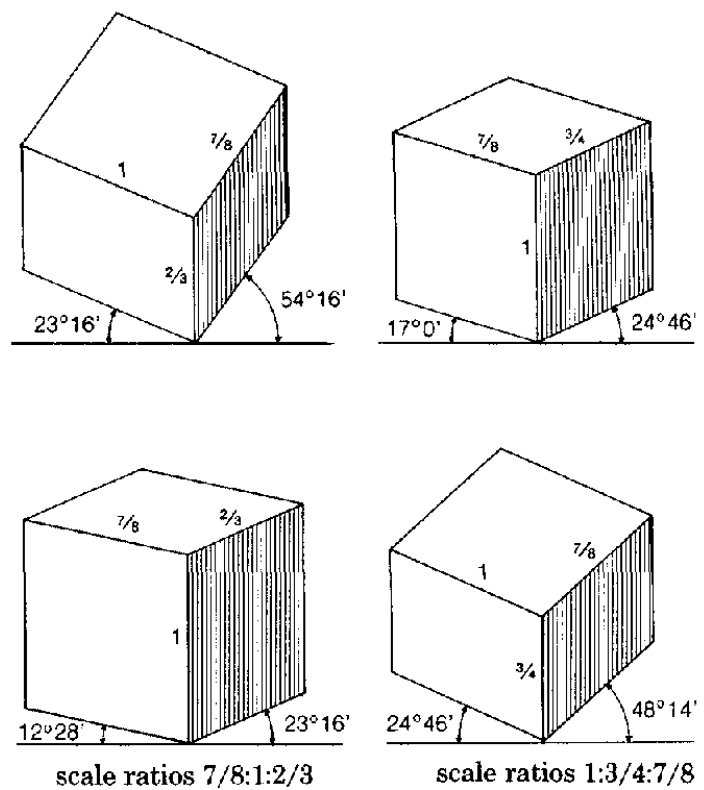

scale ratios $7 / 8: 1: 2 / 3$

Figure 3-9. Trimetric projections of a cube.

dimetric projection in Figure $3-10 \mathrm{~b}$ has reduced the top area of the bookcase, giving more emphasis to the front and side, whereas the dimetric projection in Figure $3-10 \mathrm{c}$ has reduced the side area, giving more emphasis to the largest face of the bookcase in order to lessen the distortion of this face. A trimetric projection allows almost complete freedom in choice of projection plane, and, if the plane is properly chosen, gives the most realistic appearance. A trimetric view of the bookcase is illustrated in Figure 3-10d.

\subsection{Oblique Projections}

Oblique projections combine properties of multiview orthographic and axonometric projections. A multiview orthographic projection illustrates the exact shape of two or more faces of an object. Such a representation has the disadvantage, however, that the three-dimensional shape of the object may be hard to visualize from the separate views. The axonometric projections describe the general three-dimensional appearance of an object in one view, but they rarely show the true shape of any face of an object, and measurements can be made to scale only in the directions of the projected principal axes. An oblique projection usually presents the exact shape of one face 


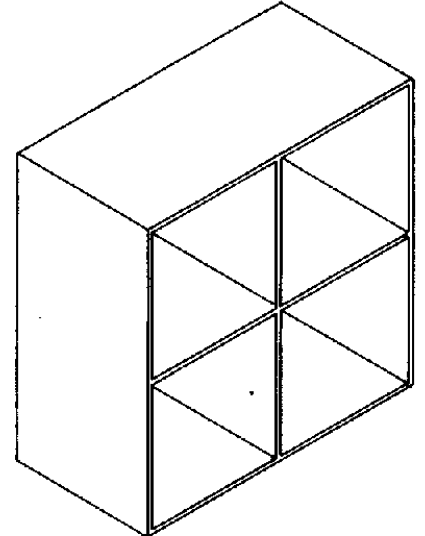

FIgURE 3-10a. Isometric projection of a bookcase.

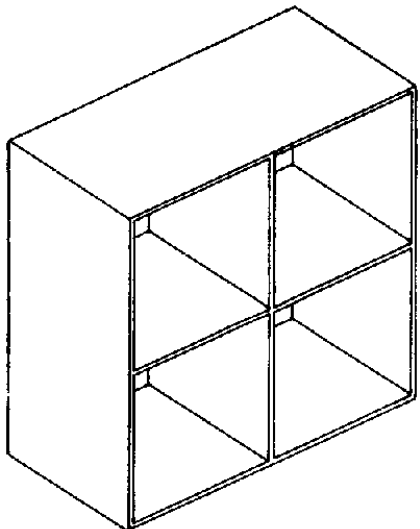

Figure 3-10c. Dimetric projection of a bookcase. The side face is reduced in size.

of an object, and at the same time illustrates its general three-dimensional appearance.

The orthographic projections are characterized by projectors that are perpendicular to the projection plane. These projections are therefore completely determined by the orientation of the projection plane. An oblique projection is characterized by projectors that are at an oblique angle to the projection plane and is determined by:

1) the orientation of the projection plane,

2) the angle between the projectors and the projection plane, and

3) the orientation of the projectors about the projection plane normal.

The projection plane of an oblique projection is usually positioned parallel either to the largest principal face of the object or to the principal face with most detail, so that this face is projected without distor-

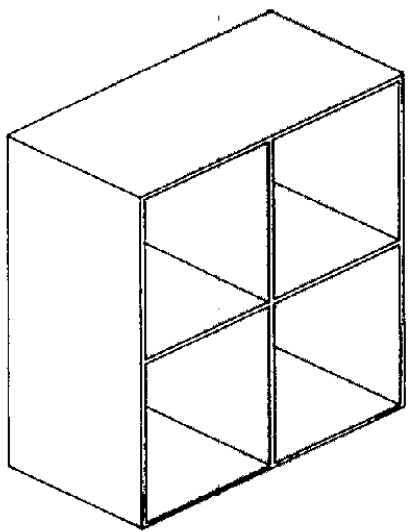

Figure 3-10b. Dimetric projection of a bookcase. The top face is reduced in size.

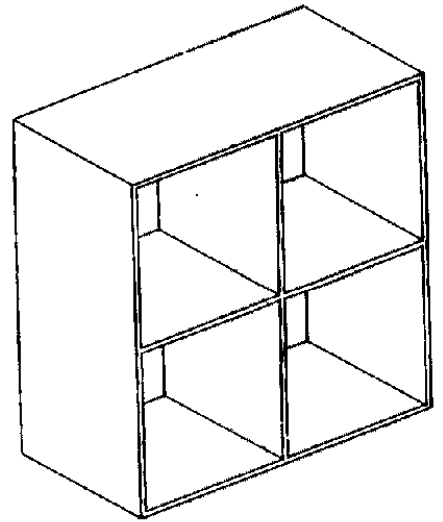

Figure 3-10d. Trimetric projection of a bookcase.

tion. The projectors are chosen to best illustrate the third dimension.

An oblique projection is classified by the angle between the projectors and the projection plane. If the angle is $45^{\circ}$ the projection is cavalier; if the angle is $\operatorname{arccot}(1 / 2)$, which is approximately $64^{\circ}$, the result is a cabinet projection. The angle between the projectors and the projection plane determines the foreshortening ratio of lines perpendicular to the projection plane. A cavalier projection results in perpendiculars projected at full scale, i.e., without foreshortening, whereas a cabinet projection gives foreshortening of one-half. The mathematical relationships between the angle and the foreshortening ratios are illustrated in Section A.6 of the Appendix. A cube, the projection plane, the projectors, and the projected cube are illustrated in Figure 3-11.

The choice of projectors determines how realistically the third dimension is repre- 


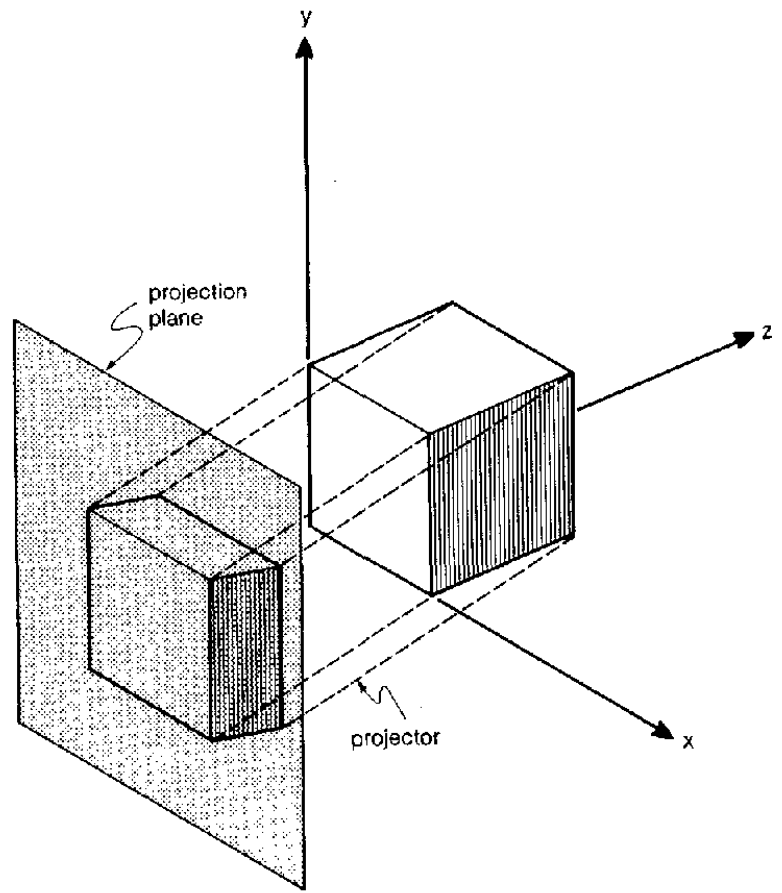

Figure 3-11a. Construction of an oblique projection.

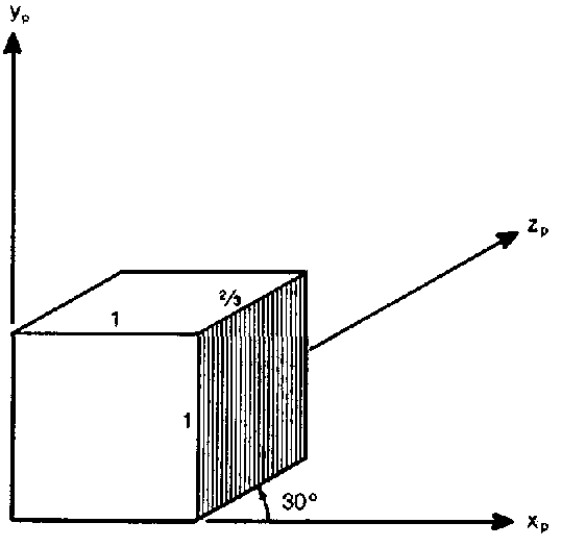

Figure 3-11b. Oblique projection resulting from the construction in Figure $3-11 a .\left(x_{p}, y_{p}\right.$ and $z_{p}$ are the projected coordinate axes.)
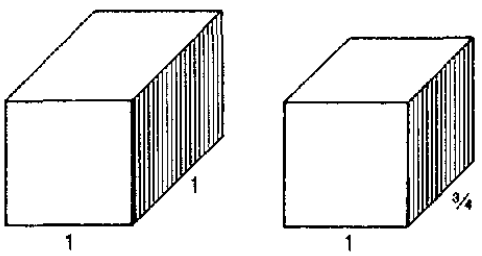

sented. The angle between the projectors and the projection plane determines the "thickness" of the projected object, and the orientation of the projectors with respect to the projection plane normal determines the relative emphasis of the receding planes.

The proportions of a cavalier projection often seem distorted-objects appear too thick. Similarly, objects represented by a cabinet projection sometimes seem too thin. These projections are used for ease of measurement, but foreshortening ratios of $2 / 3$ or $3 / 4$ may give more pleasing views. Figure 3-12 illustrates a cube projected with foreshortening of one, three-fourths, twothirds, and one-half, respectively.

The proportions of an oblique view can also be varied by changing the orientation
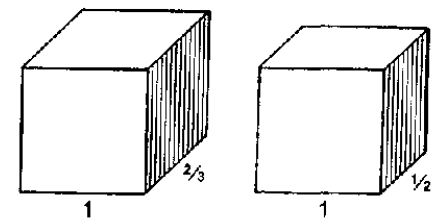

Figure 3-12. Oblique projections of a cube. The foreshortening ratio varies with the angle between the projectors and the projection plane. 

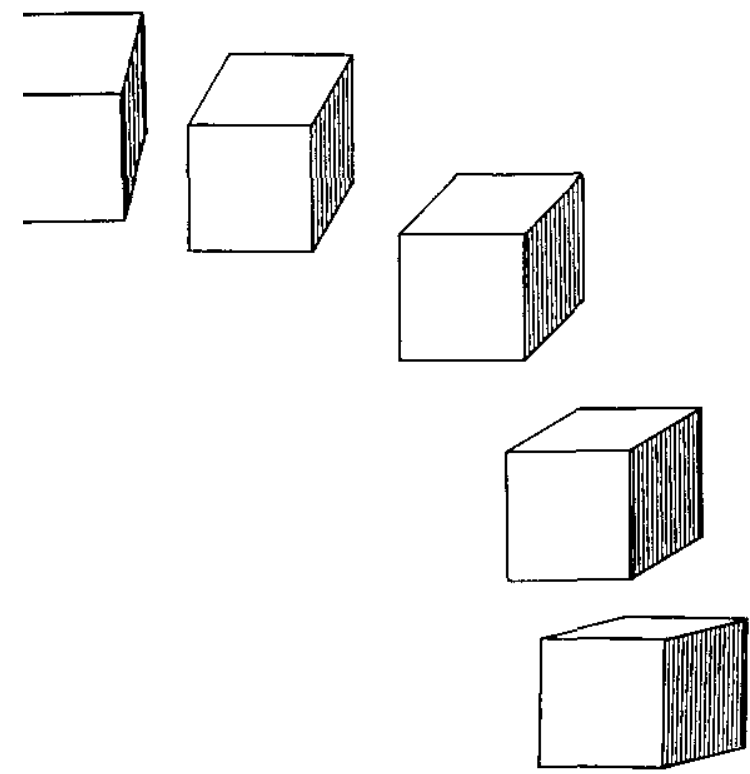

Frgure 3-13. Elevation oblique projections of a cube. The vertical face is shown at true shape, and the relative emphasis of the receding faces varies with the orientation of the projectors. of the projectors. One of the receding planes can be emphasized and the other de-emphasized. This is demonstrated in Figures 3-13 and 3-14. Figure 3-13 illustrates elevation oblique, i,e., with the vertical faces shown at true shape. Figure 3-14 illustrates plan oblique, in which the horizontal faces of the cubes are represented at true shape.

A projector is best defined with respect to a coordinate system with two axes in the projection plane and the third along the projection plane normal. The angle between the projector and the projection plane and the angle of rotation of the projector about the normal are two spherical coordinates of the projector with respect to this coordinate system. This relationship is illustrated in Section A.6 of the Appendix. For cavalier and cabinet projections a rotation of the projector about the normal is often chosen such that the projection plane normal is projected at $30^{\circ}$ or $45^{\circ}$ with respect to the horizontally projected coordinate axis. (One way of defining this coordinate system is shown in Section 4.)
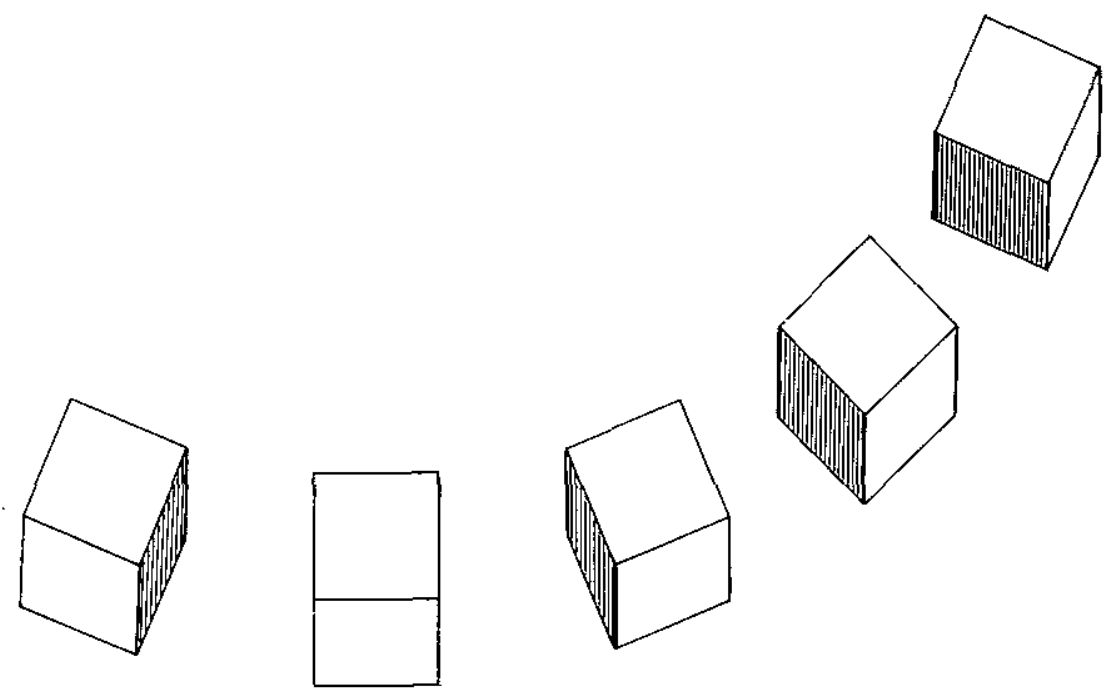

Figure 3-14. Plan oblique projections of a cube. The horizontal face is shown at true shape, and the relative emphasis of the receding faces varies with the orientation of the projectors. 

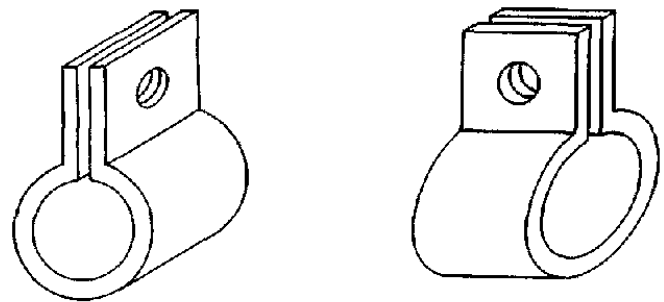

Figure 3-15. a) Oblique projection chosen according to rule 1. b) Oblique projection chosen in violation of rule 1 .

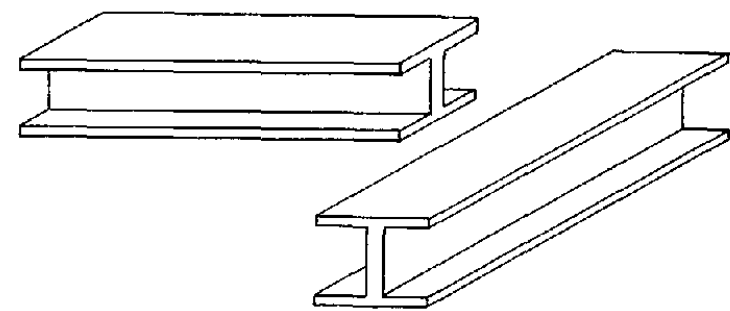

Figure 3-16. a) Oblique projection chosen according to rule 2. b) Oblique projection chosen in violation of rule 2 .

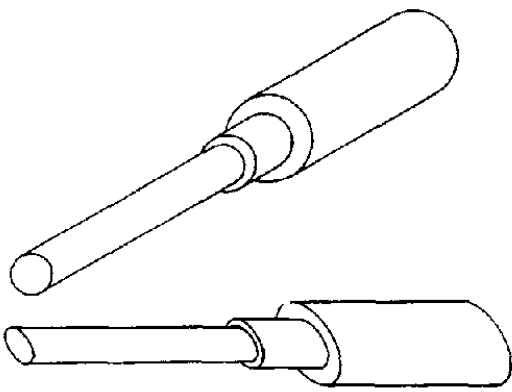

Figure 3-17. Oblique projections showing that rule 1 should take precedence over rule 2.

The shape of an object determines how to choose the projection plane for an oblique projection. In order to minimize distortion and to provide a more realistic appearance, the projection plane should be chosen so that:

1) it is parallel to the most irregular of the principal faces or to the one which contains circular or curved surfaces; or

2) it is parallel to the longest principal face of the object.

The projection in Figure 3-15a gives a less distorted view than that in Figure 315b. Similarly, the projection in Figure 316a seems less distorted than that in Figure $3-16 \mathrm{~b}$. When these rules conflict the first should generally prevail over the second, as is illustrated in Figure 3-17.

\subsection{Perspective Projections}

A perspective projection gives a natural appearance of an object as seen by the eye. However, such a projection does not preserve the shape of an object, and measurements can be made to scale only in the parts of the object that lie in the projection plane.

A perspective projection is distinguished from a parallel projection by:

1) convergence of parallel lines,

2) diminution of size, and

3) nonuniform foreshortening.

Only lines parallel to the projection plane remain parallel in a perspective view. Parallel lines that are not parallel to the projection plane converge to a single point, called a vanishing point. The vanishing point for a set of parallel lines is the point where a line through the center of projection, parallel to the set of parallel lines, intersects the projection plane. A principal vanishing point is a vanishing point of a principal axis. Vanishing points for lines parallel to a plane always lie along a straight line in the projection plane. When this line appears horizontal to the observer,

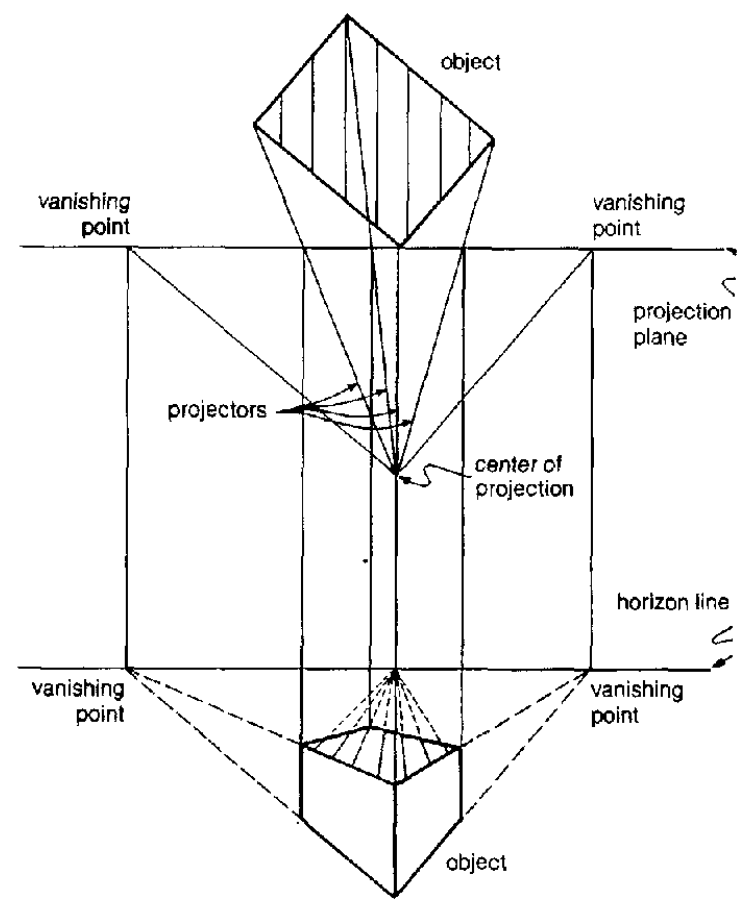

Figure 3-18. Plan view of perspective construction and the resulting perspective projection. 


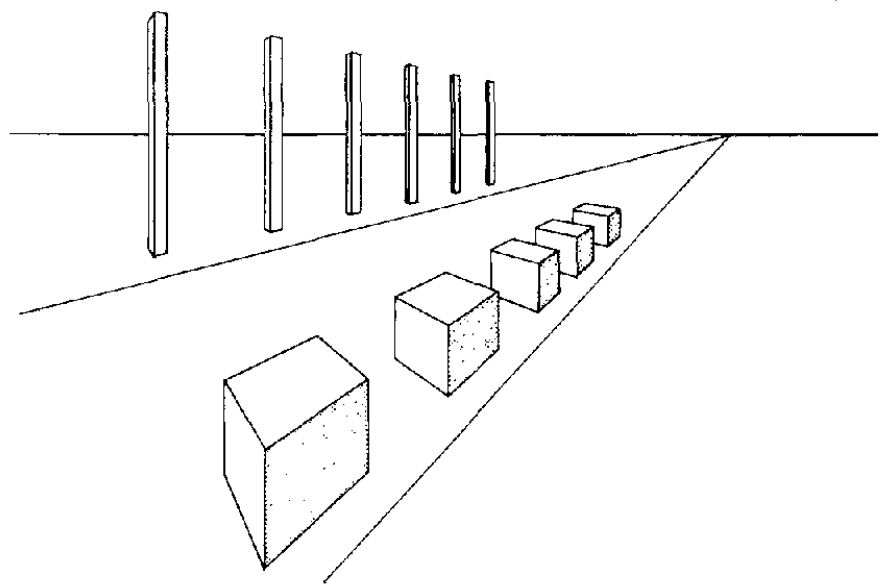

Figure 3.19. Perspective projection. Diminution.

it is referred to as the horizon line. The top half of Figure 3-18 is a plan view of an object, a projection plane, the center of projection, and the projectors. The bottom half illustrates the corresponding perspective view of the object, the vanishing points, and the horizon line.

The convergence of parallel lines results in both diminution of size and in nonuniform foreshortening of objects. Objects of equal size appear smaller as their distance from an abserver increases and become larger as that distance decreases. Only areas in the projection plane retain their true size. Figure 3-19 illustrates the diminution of equal-sized objects when they are placed farther away from the observer.

The shape of an object is rarely preserved under a perspective projection. Parallel lines are unequally foreshortened depending on their position relative to the observer. Circles generally project to ellipses. Only the parts of an object parallel to the projection plane retain their shape. Nonuniform foreshortening of parallel lines and circles is illustrated in Figure 3-20.

A perspective projection of an object is determined by five variables:

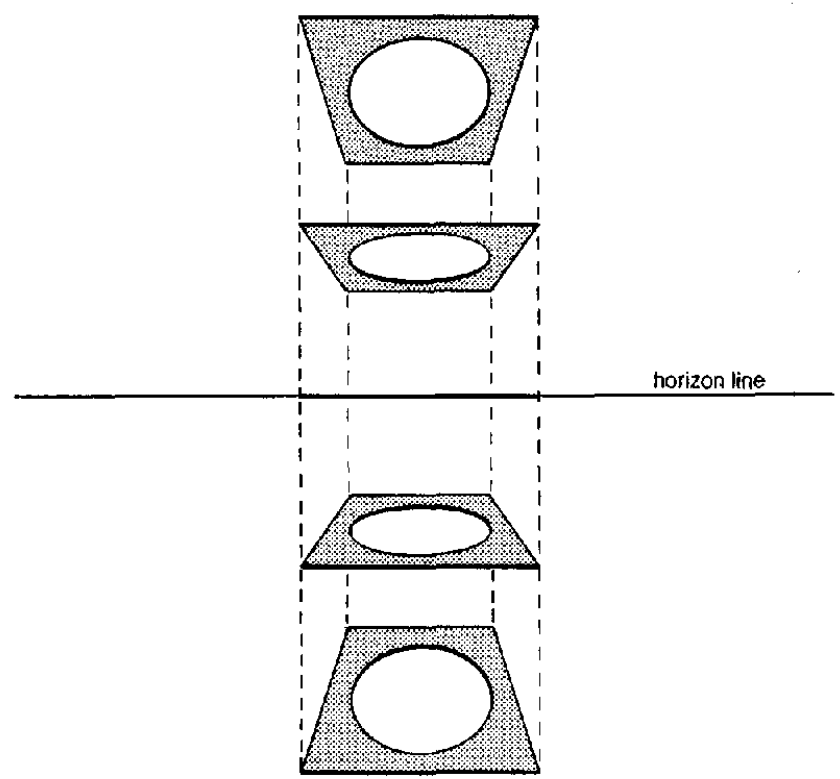

FIgURE 3-20. Perspective projection. Non-uniform foreshortening. 

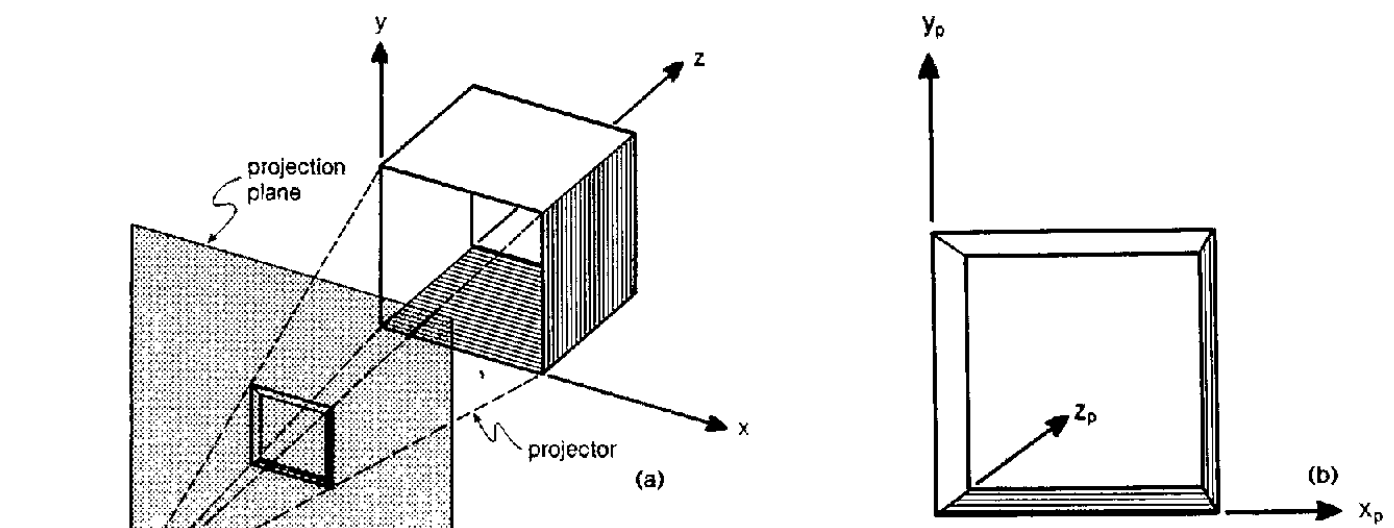

Figure 3-21. a) Construction of a one-point percenter of projection

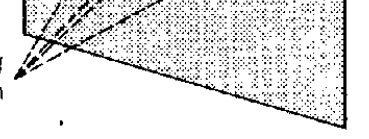
spective projection. b) One-point perspective projection resulting from the construction in $a) .\left(x_{p}, y_{p}\right.$ and $z_{p}$ are the projected coordinate axes.)

1) the orientation of the projection plane with respect to the object (i.e., the angles between the projection plane and the principal coordinate axes),

2) the height of the center of projection with respect to the object (i.e., the position of the horizon line with respect to the object),

3) the distance of the center of projection to the object,

4) the distance of the projection plane to the object, and

5) the horizontal displacement of the center of projection relative to the center of the object.

The values of these five variables are generally chosen so as to give the most realistic appearance of the object.

The perspective projections are classified according to the number of principal coordinate axes that intersect, but do not lie within, the projection plane. As is shown in Section A.7 of the Appendix, this is equal to the number of finite principal vanishing points.

A one-point (or parallel) perspective projection is the type of projection in which the projection plane intersects only one of the principal coordinate axes. Hence, to obtain a one-point perspective, the projection plane must be parallel to one of the principal planes. A cube, along with the projection plane, the projectors, and the one-point perspective of the cube, is illustrated in Figure 3-21.

A two-point (or angular) perspective pro- jection is the type of projection where the projection plane intersects two of the principal coordinate axes. A two-point perspective is obtained by choosing the projection plane parallel to one of the principal axes, but not parallel to any coordinate plane. A two-point perspective of a cube is illustrated in Figure 3-22.

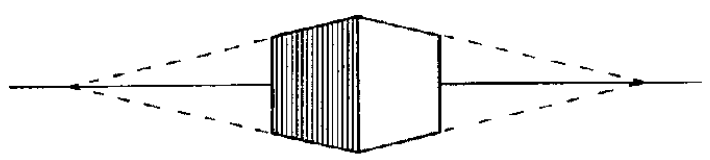

Figure 3-22. Two-point perspective projection.

A three-point perspective projection is the type of projection where the projection plane intersects all of the coordinate axes. A three-point perspective is obtained by choosing the projection plane so that it is not parallel to any coordinate axis. A threepoint perspective of a cube is illustrated in Figure 3-23.

The five variables listed above control the pictorial effects of a perspective projection, and if not chosen properly may produce unpleasant distortions. If a perspective projection is to illustrate an object as it is seen by an observer, the choice of station point $t^{11}$ and position of the projection plane are fairly limited. Certain distortions are, however, at times acceptable or even desirable to obtain more interesting

\footnotetext{
"This term is commonly used in architectural drawing to indicate the position of the viewer, and is equivalent to the center of projection.
} 


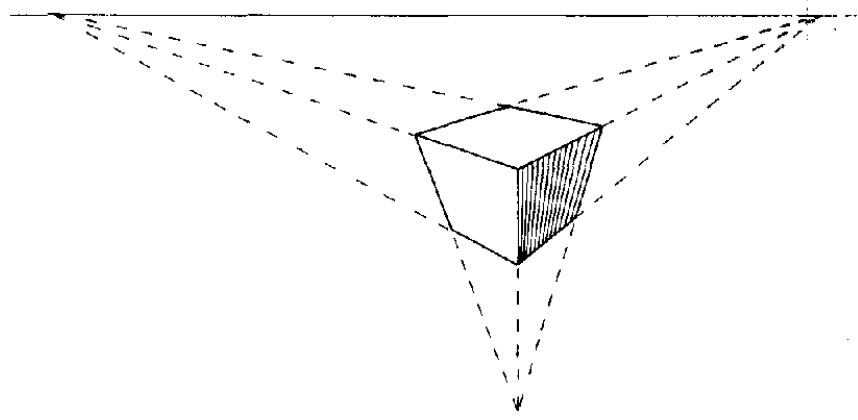

Figure 3-23. Three-point perspective projection.

and dramatic illustrations. The effects of these variables on the three types of perspective are discussed below.

In general, the station point should be selected at a position from which an object would be viewed "naturally." The distance from the station point to the object should be such that the object is well within the observer's field of view with the horizon line near the observer's eye level. The station point should not be displaced horizontally too far off-center, and it should be opposite the center of interest in the picture. As was illustrated in Piero's Resurrection (Figure 1-3), the viewer's attention is naturally drawn to the area in the painting opposite the station points. (The reader should note that this painting is a study in one-point perspective. It was painted from two vantage points, and hence has two principal vanishing points.)

In a one-point perspective, the projection plane is parallel to one of the major faces of the object, that is, the observer is looking straight at this face. A one-point perspective has the advantage that it may show three adjacent vertical faces, one at true shape. A one-point perspective is used mostly for interior spaces, street scenes, and objects such as pieces of furniture.

The orientation of the projection plane cannot be varied in a one-point perspective. The observer is always looking at one face, which gives a symmetric view that sometimes appears dull and static. Moving the center of projection slightly off-center horizontally may produce a more interesting perspective. This situation is illustrated in Figure 3-24. One of the side walls is empha- sized more than the other, and the viewer's attention is naturally drawn to the center of interest, which is to the side of the center

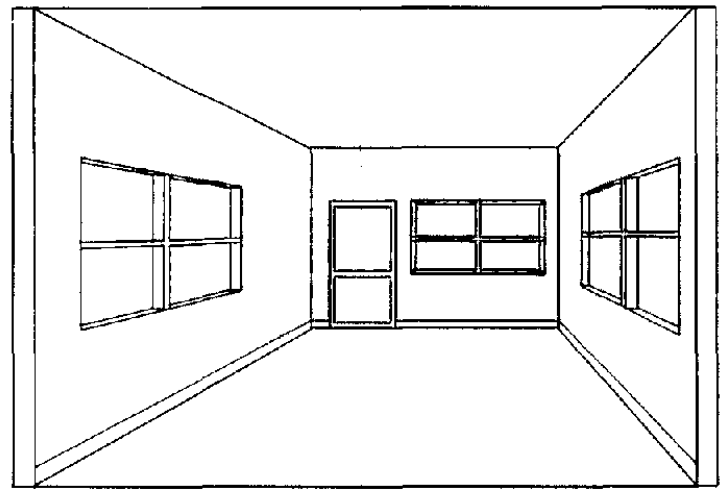

Figure 3-24. One-point perspective projection with station point off-center.

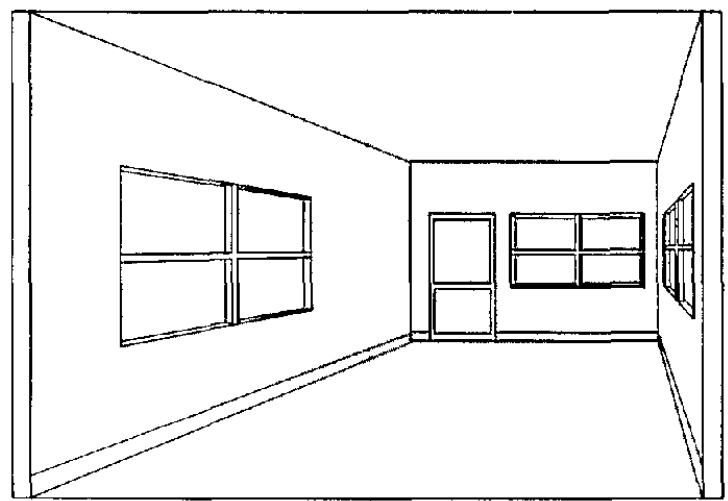

Figure 3-25. One-point perspective projection with station point too far off-center. 
of projection. If the center of projection is moved too far off-center, an objectionable view may result, as is illustrated in Figure 3-25. The distortion in Dürer's St. Jerome in His Study (Figure 1-6) is due mostly to this phenomenon. (A nonsymmetric view with heavy emphasis on one of the side walls would generally be better illustrated with two-point perspective.)
Moving the horizon line up and down changes the proportions of ceiling and floor in an interior view (Figure 3-26), or shows the exterior view of a building as if it were seen from above or below (Figure 3-27). An indoor view should generally have the horizon line at the eye-level of a sitting or standing observer. For exterior views a more interesting projection is often

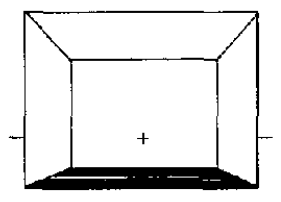

horizon near floor

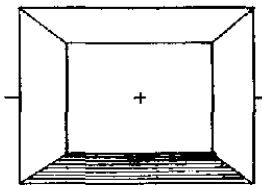

horizon centered

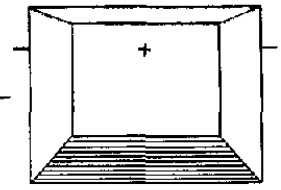

horizon near ceiling

Figure 3-26. One-point perspective projections of interior of a room with different heights of horizon line.

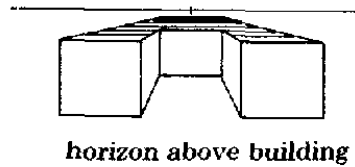

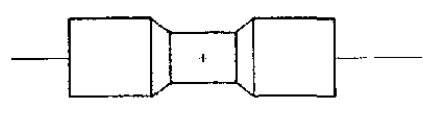

horizon centered

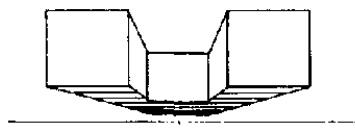

horizon below building

Figure 3-27. One-point perspective projections of exterior of a building with different heights of horizon line.
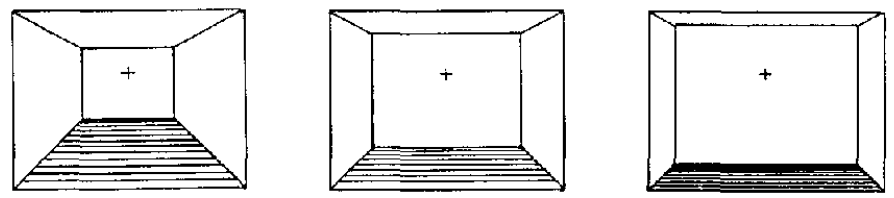

station point close to room

station point at medium distance

station point far from room

FIGURE 3-28. One-point perspective projections of interior of a room with different distances from station point to room.

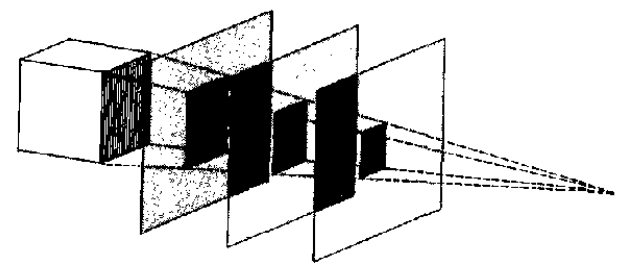

Figure 3-29. The distance from the plane to the station point affects only the size of a perspective projection. 
achieved with the horizon above or below the object.

The distance of the station point to the object determines whether it is viewed from far or near. When the station point is too close to the object, the depth in the projection seems exaggerated. If it is too far away from the object, the projected object seems flat. The effect of distance is illustrated in Figure 3-28. The station point should be chosen at a distance from an object at which an observer would naturally view the object, so as to include the entire object in the field of vision.

Figure 3-29 shows how moving the projection plane with respect to the station point scales the perspective projection uni- formly. Zooming can be implemented by slowly moving the projection plane towards or away from the station point.

In a two-point perspective the projection plane is parallel to one of the major axes of the object, i.e., the observer is looking straight at one edge of an object but not straight at any of the faces adjacent to this edge. Unlike a one-point perspective, a twopoint perspective does not generally give a symmetric view. For this reason, it is more widely used.

In a two-point perspective, the orientation of the projection plane with respect to the object can be varied so as to emphasize one vertical face or the other. In Figure 330 the proportions of the left and right side

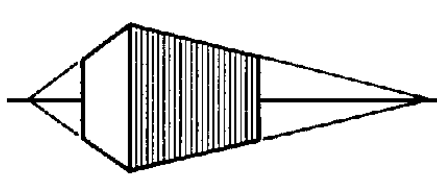

right wall emphasis

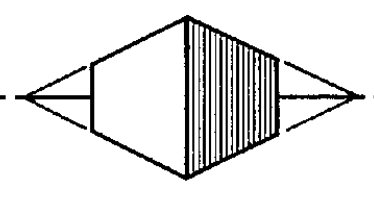

equal wall emphasis

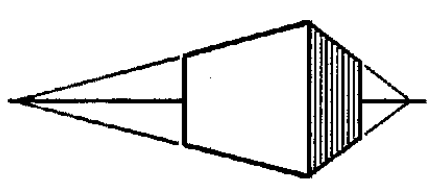

left wall emphasis

Figune 3-30. Two-point perspective projections with different orientations of projection plane and positions of station point.

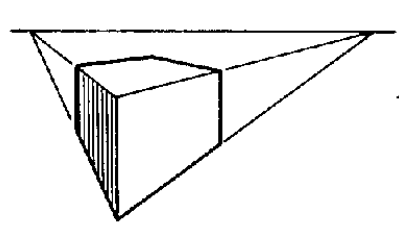

horizon above object

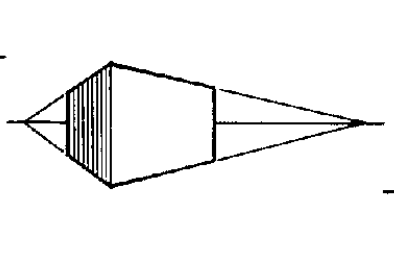

horizon centered

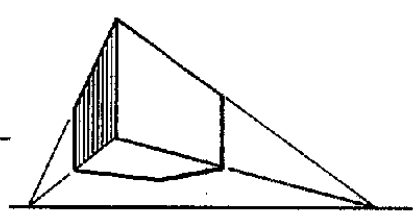

horizon below object

FIgure 3-31a. Two-point perspective projections with different heights of horizon line.

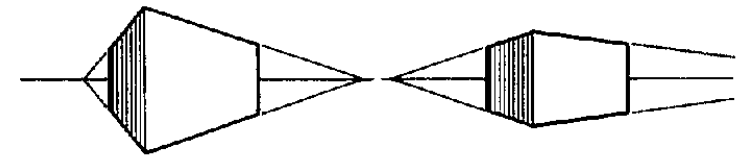

station point close to object station point at medium distance

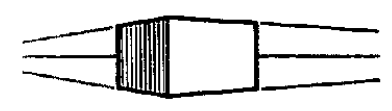

station point far from object

FIgURE 3-31b. Two-point perspective projections with different distances from station point to object.

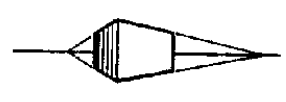

projection plane far from object

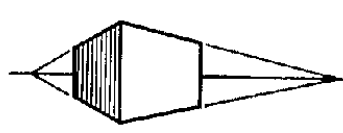

projection plane at medium distance

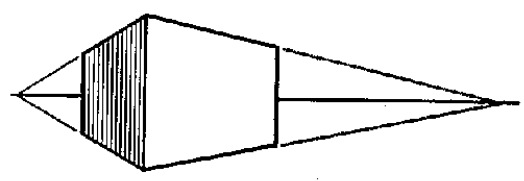

projection plane close to object

FrGuRE 3-31c. Two-point perspective projections with different distances from projection plane to object. 


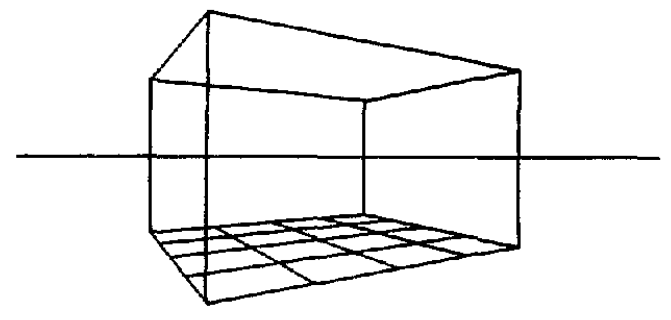

station point at natural distance

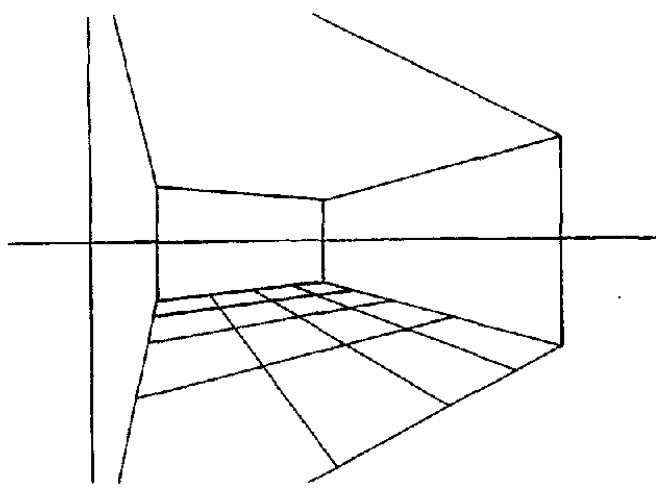

station point too close

Figure 3-32. Two-point perspective projections. Distortion of regular patterns.

of an object are varied by changing the orientation of the projection plane. In each drawing the station point is located on a normal of the projection plane, through the vertical center of the object. As for a onepoint perspective, the station point can be moved off-center horizontally to draw the viewer's attention to one side of the drawing. Care should be taken when positioning the station point off-center, since this may produce distortions whose cause is not as easily detectable as those for a one-point perspective.

By varying the position of the horizon line, the distance from the station point to the object, and the position of the projection plane relative to the object, effects similar to those described for one-point perspective can be obtained. These variations are illustrated in Figure 3-31.

As for one-point perspective, the station point should be chosen at a position from which an observer would naturally view the object. If the station point is too close to an object, unpleasant distortion can occur, particularly at the edges of the projected object. This is especially noticeable for ob- jects with regular shapes and with repetitive patterns as is illustrated in Figure 3-32. To avoid such distortions it is generally recommended that the viewed object be within a $45^{\circ}-60^{\circ}$ cone of vision. A station point close to an object can, however, sometimes cause interesting effects. Although a person inside a room generally sees only what is within a narrow field of view, he is conscious of objects in an almost $180^{\circ}$ field of vision. Including objects outside the $60^{\circ}$ cone of vision gives the viewer a feeling of being inside a room, rather than seeing it from a distance. This illusion is illustrated in Figure 3-33.

In a three-point perspective, the projection plane is inclined to all three principal faces of the viewed object. The effects are, for example, those seen by an observer looking up at a tall building, or looking down from its roof. Changes in the five variables result in changes to the threepoint perspective that are similar to, but
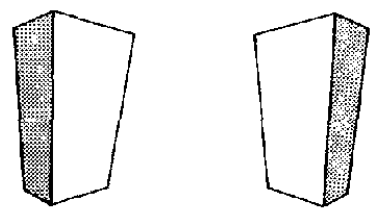

right wall emphasis left wall emphasis

Figure 3-34a. Three-point perspective projections of a building as seen from one side or the other.
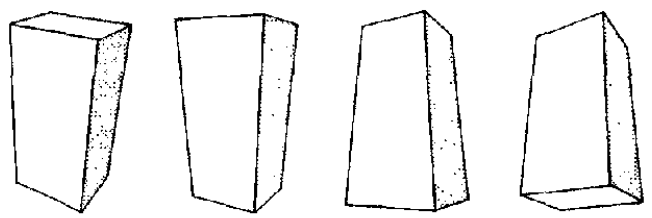

station point station point station point station point above building near top near bottom below building

Figure 3-34b. Three-point perspective projections of a building as seen from different heights.

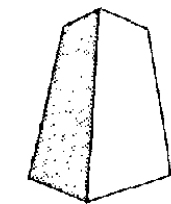

station point close to building

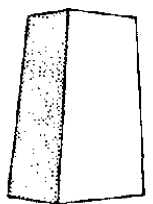

station point far from building
Figure 3-34c. Three-point perspective projections of a building as seen from different distances. 


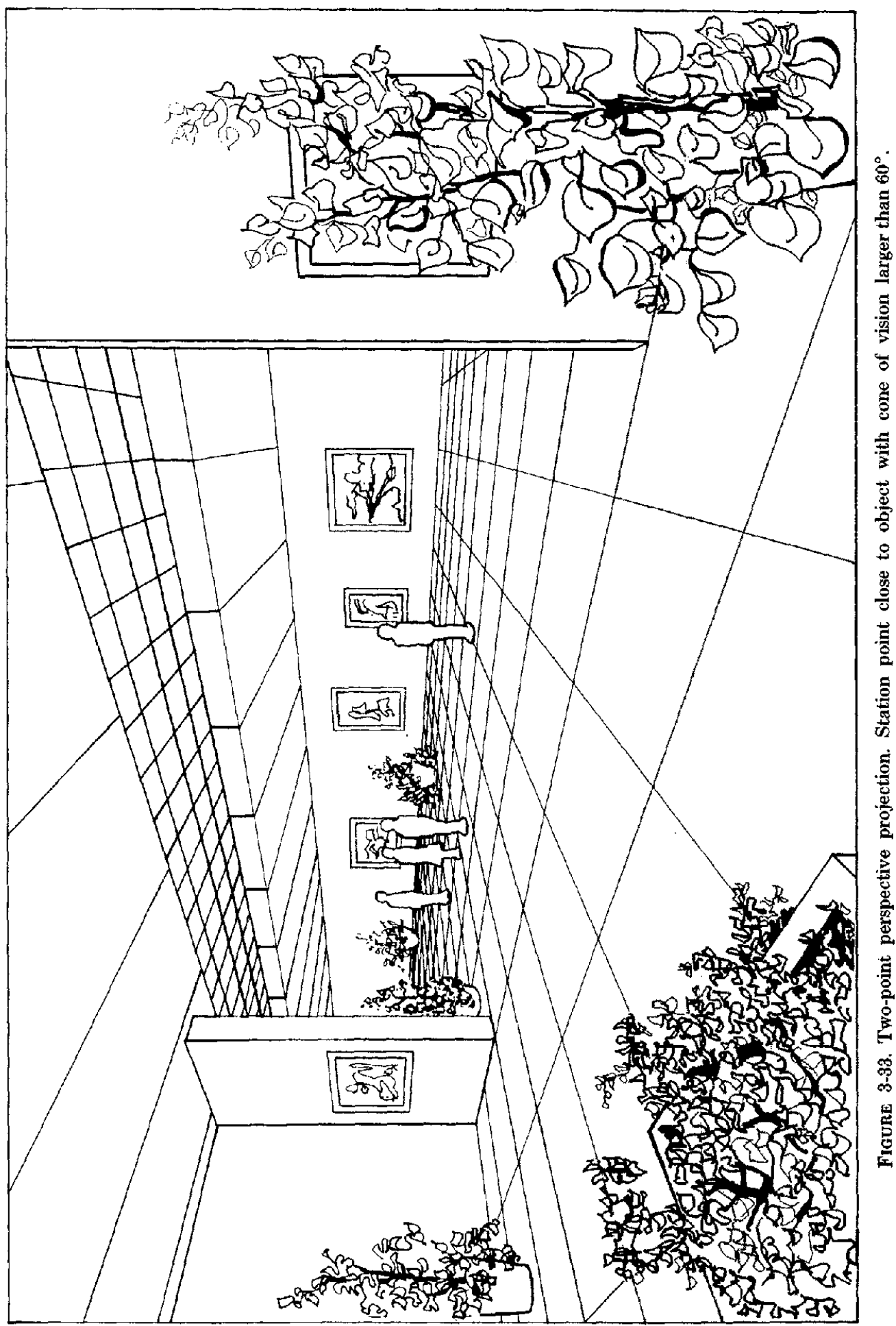


more dramatic than, those for both onepoint and two-point perspective.

The orientation of the projection plane determines both the proportions of the vertical faces and the rate at which vertical lines converge. Figures 3-34a and 3-34b illustrate the effects obtained by looking at an object from one side or another, and by looking at it from above or below, respectively. This is accomplished by rotating the projection plane vertically and horizontally, and keeping the station point on a projection plane normal through the center of interest on the object. The effect obtained by changing the distance of the station point to the object is illustrated in Figure $3-34 \mathrm{c}$.

\section{SPECIFICATIONS OF VIEWING TRANSFORMATIONS IN A GRAPHICS SYSTEM}

A viewing transformation defines a mapping of a three-dimensional object onto a two-dimensional display surface. In addition to the specification of a projection, the viewing transformation also defines the portion of the object that is to be viewed and a mapping of the projected image to the display surface. This section will discuss a set of constructs that allows the specification of planar geometric projections in a graphics system. For a general discussion of viewing transformations the reader is referred to NewM73, GSPC77, and BERg78.

Most contemporary graphics subroutine packages provide only limited capabilities for defining projections of three-dimensional objects. In order to get a desired view, the programmer must often rotate, translate, scale, and shear the object before mapping it to the projection plane. This section describes a set of constructs that is sufficiently general to specify a projection only in terms of a projection plane and a center of projection. This method of specification is suitable for a general purpose graphics package because it allows consistent specifications of parallel and perspective projections, and because few constructs are needed to specify a projection.

There are alternate ways of defining planar geometric projections. For example, an orthographic projection could be speci- fied by the foreshortening ratios of the principal axes, and an oblique projection by the foreshortening ratios of the principal axes and the angle of the receding axis. A perspective projection could be specified by the eye position of the viewer and the three principal vanishing points. These methods of specification are conceptually simpler for many applications.

The constructs used in this section have equivalent viewing functions in the Core Graphics System. Section A.9 of the Appendix briefly introduces these viewing functions and gives a few programming examples. For a full discussion of the Core Graphics System, the reader is referred to GSPC77 and to BERG78.

A projection plane can be defined by one point (here called the reference point) and a vector that defines a normal of the plane. As will be seen below, it is sometimes convenient to position the plane at a distance from the reference point. The projection plane is positioned perpendicular to the normal, at a specified distance from the reference point.

The direction that is "up" in the projection plane is specified by another vector. This vector and the projection plane normal determine a new coordinate system referred to as the UV-system. The V-axis is the orthographic projection of the given vector in the projection plane; the $\mathrm{U}$-axis is the cross-product of the projection plane normal with the $V$-axis. The $V$-axis will be vertical on the display surface, and the $U$ axis horizontal.

The center of projection is defined by a vector. For a parallel projection, the center of projection is at infinity and the vector defines the direction of the projectors. For a perspective projection the vector defines the position of the center of projection relative to the reference point.

The remainder of this section gives some examples of how to position the center of projection and the projection plane to obtain various views of an object for each type of projection. In particular, it illustrates how these constructs are used to obtain certain desired foreshortening ratios for parallel projections, and to obtain a sequence of perspective views of an object as it would be seen by a moving observer. 
A parallel projection is orthographic when the projectors are normal to the projection plane. Therefore, when the direction of the projectors is collinear with the projection plane normal, an orthographic projection results. Hence, an orthographic projection is simply defined by the position of the projection plane. ${ }^{12}$ For all parallel projections it is often convenient to position this plane at the origin, that is, to position the reference point at the origin and to choose the distance to the plane equal to zero. $^{13}$

A principal view of a multiview orthographic projection is obtained by positioning the projection plane parallel to one of the principal coordinate planes. This is accomplished by specifying a projection plane normal perpendicular to one of these planes. Similarly, an auxiliary view is obtained by specifying a normal perpendicular to the face of the object that should be projected without distortion.

An axonometric view is obtained by choosing a plane that is not parallel to any coordinate plane. As was illustrated in Section 3, an isometric projection is an axonometric projection with the projection plane normal parallel to one of the lines $\pm x= \pm y$ $= \pm z$. Hence, if the absolute values of the components of the normal are all equal, an isometric projection is obtained. Similarly, if exactly two of the absolute values of the components of the normal are equal, a dimetric projection results.

Given the foreshortening ratios of the principal axes, the direction of the projection plane normal can easily be calculated. As is demonstrated in Section A.5 of the Appendix, the foreshortening ratio, $l$, is defined by $l=\cos \theta$, where $\theta$ is the angle between the coordinate axis and the projection plane. But this angle is the complement of the angle between the normal and the coordinate axis. The direction of the normal can be defined by its direction cosines, $\left(n_{1}\right.$, $\left.n_{2}, n_{3}\right)$, where

$$
n_{1}=\cos \left(90^{\circ}-\theta\right)=\sin \theta
$$

\footnotetext{
$\overline{12}$ As in the previous section, it is assumed that the object is oriented with its principal axes along the principal coordinate axes.

${ }^{13}$ This does not take into account sectional views and hidden line processing.
}

The values for $n_{2}$ and $n_{3}$ are calculated in the same manner.

The projection plane for an oblique projection is chosen parallel to the face of the object that should be projected without distortion. The projection direction is no longer collinear with the projection plane normal, but at an angle to this normal.

The projector is best defined with respect to the coordinate system defined by $U, V$, and the projection plane normal. As was discussed in the previous section, the angle between the projector and the projection plane and the angle of rotation of the pro. jector about the normal are two spherical coordinates of the projector with respect to this coordinate system. The Cartesian coordinates of the projectors can be calculated from its spherical coordinates or from the foreshortening ratio, $l$, and the angle $\gamma$ between the receding principal axis and the U-axis. As is demonstrated in Section A.6 of the Appendix, the direction of the projectors is $(l \cos \gamma, l \sin \gamma,-1)$.

A perspective projection is defined by a projection plane and a center of projection. The choice of reference point depends on the application. It can be chosen at the station point if, for example, one purpose of the application is to illustrate what is in a moving observer's field of view. The reference point may also be chosen on or near the object. This makes it easy to alter the values of the five variables that determine the appearance of a perspective view.

To create a one-point perspective projection, the reference point can be chosen in the center of the face of the object that is to be parallel to the projection plane. The center of projection is easily determined so that the distance to the object insures that it is within a $45^{\circ}-60^{\circ}$ cone of vision, that the horizon line is at the eye height of the observer, and that the horizontal displacement gives a desirable view. By varying only the position of the center of projection relative to the reference point, the visual effects can easily be changed.

To create a two-point perspective, the reference point can be chosen on one vertical edge of the object. The projection plane normal will determine the relative emphasis between the two adjoining faces. The center of projection is determined, 
much the same way as for one-point perspective, to create a desired visual effect.

The reference point may also be chosen at the eye-pont of the observer, i.e., at the center of projection. The projection plane normal is then defined by a vector from the eye-point to the center of interest on the object, and the projection plane at some given distance from the eye-point. By changing the reference point and the projection plane normal, one can illustrate what is in the observer's field of view as he moves about. Note, however, that when the center of projection and the reference point coincide, the variables that determine a perspective view are not as easily modified, since the center of projection is positioned on the projection plane normal.

\section{CONCLUSION}

This paper has presented the planar geometric projections in a manner suitable for applications in computer graphics. The method of projection has been defined in terms of the position of the projection plane and the center of projection. The choice of projection plane and projectors has been related to the pictorial effects of the projected object. A simple, straightforward way to implement the projections is presented in the Appendix.
There is no hard and fast rule for choosing one type of projection over another. This choice must be made accordng to the purpose for which the projection is to be used, according to the shape of the object, and according to the pictorial effects that are to be achieved. This paper provides some guidelines for making such a choice.

\section{ACKNOWLEDGMENTS}

The authors would like to express their appreciation to the reviewers, especially Professor Wayne Dowling of Iowa State University, for their many valuable comments. The authors would also like to thank Dan Bergeron, Peter Bono, James Foley, James Michener, and Andy van Dam, members of the GSPC Core System Definition Subgroup, for their invaluable help on the many drafts of this paper. Several members of the Brown and RISD community also deserve credit for their helpful suggestions: Katrina Avery, Dick Bulterman, Read Fleming, Susan Lukesh, Steve Oles, Bob O'Neal, Ann Schulz, and Judith Wolin.

\section{APPENDIX}

The first three sections of the Appendix introduce homogeneous coordinates, direction cosines, and spherical coordinates -concepts that are used throughout the appendix. For a more complete treatment of homogeneous coordinates, the reader is referred to NEwM73. Direction cosines and

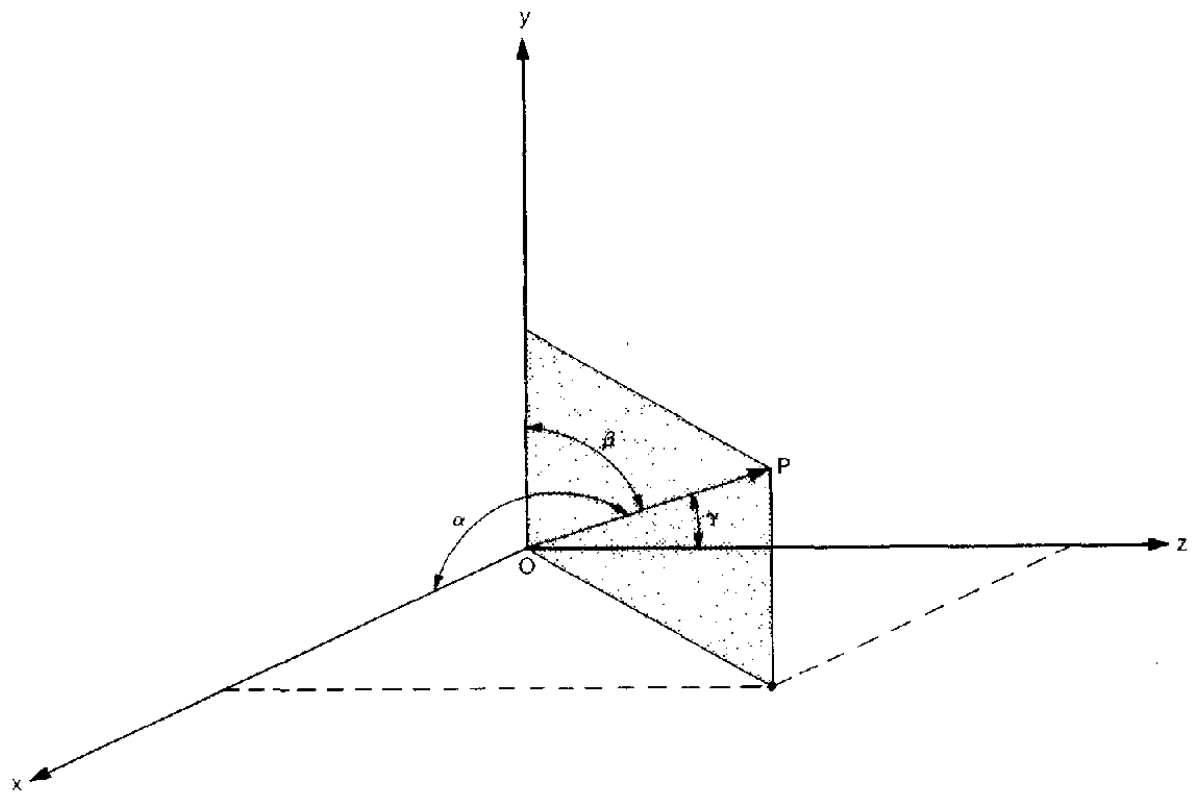

Frgure A-1. Direction cosines. 
spherical coordinates are discussed in more detail in most college-level analytic geometry textbooks.

Section A.4 derives the constraining equations for dimetric and isometric projections in terms of the pasition of the projection plane. Sections A.5, A.6, and A.7 show the relationships between the method of projection and the properties of the projected objects for axonometric, oblique, and perspective projections, respectively.

The planar geometric projections can be represented by a single $4 \times 4$ homogeneous matrix, and Section A.8 describes one possible way of deriving this matrix. The final section gives some programming examples using the viewing functions in the Core Graphics System.

\section{A.1 Homogeneous Coordinates and Matrix Representations}

The homogeneous coordinate representation of an object in $3 \mathrm{D}$ is a representation of that object in $4 \mathrm{D}$ together with a projection from 4-space to 3-space. Any point $(x, y, z)$ in $3 \mathrm{D}$ can be represented as $(x, y, z, 1)$ in $4 \mathrm{D}$. Conversely, any point $(x, y, z, w)$ with $w \neq 0$ in $4 \mathrm{D}$ represents the point $(x / w$, $y / w, z / w)$ in 3D. Thus, all points ( $h x$, $h y, h z, h w), h \neq 0$, on a line through the origin project to the same point in 3-space.
By representing a point by its homogeneous coordinates, any planar geometric projection of this point onto a plane can be expressed as a single $4 \times 4$ matrix. As will be shown in Section A.8, this matrix can be derived by composing $4 \times 4$ matrices representing three-dimensional rotation, shearing, translation, and perspective transformations. Rotation and shearing are linear transformations in $3 \mathrm{D}$ and can be expressed as $3 \times 3$ matrices. Translation and perspective, however, can only be expressed as $4 \times 4$ matrices.

A general linear transformation in 3space can be expressed as the $4 \times 4$ matrix $^{14}$

$$
\left[\begin{array}{cccc}
a_{11} & a_{12} & a_{13} & 0 \\
a_{21} & a_{22} & a_{23} & 0 \\
a_{31} & a_{32} & a_{33} & 0 \\
0 & 0 & 0 & 1
\end{array}\right]
$$

where a matrix $\left[a_{i j}\right]$ is a linear transformation. More generally, in a $4 \times 4$ matrix of the form

$$
\left[\begin{array}{cccc}
a_{11} & a_{12} & a_{13} & p_{1} \\
a_{21} & a_{22} & a_{23} & p_{2} \\
a_{31} & a_{32} & a_{33} & p_{3} \\
t_{1} & t_{2} & t_{3} & 1
\end{array}\right]
$$

${ }^{14}$ Note that a point is represented as a row vector which is post-multiplied by the matrices.

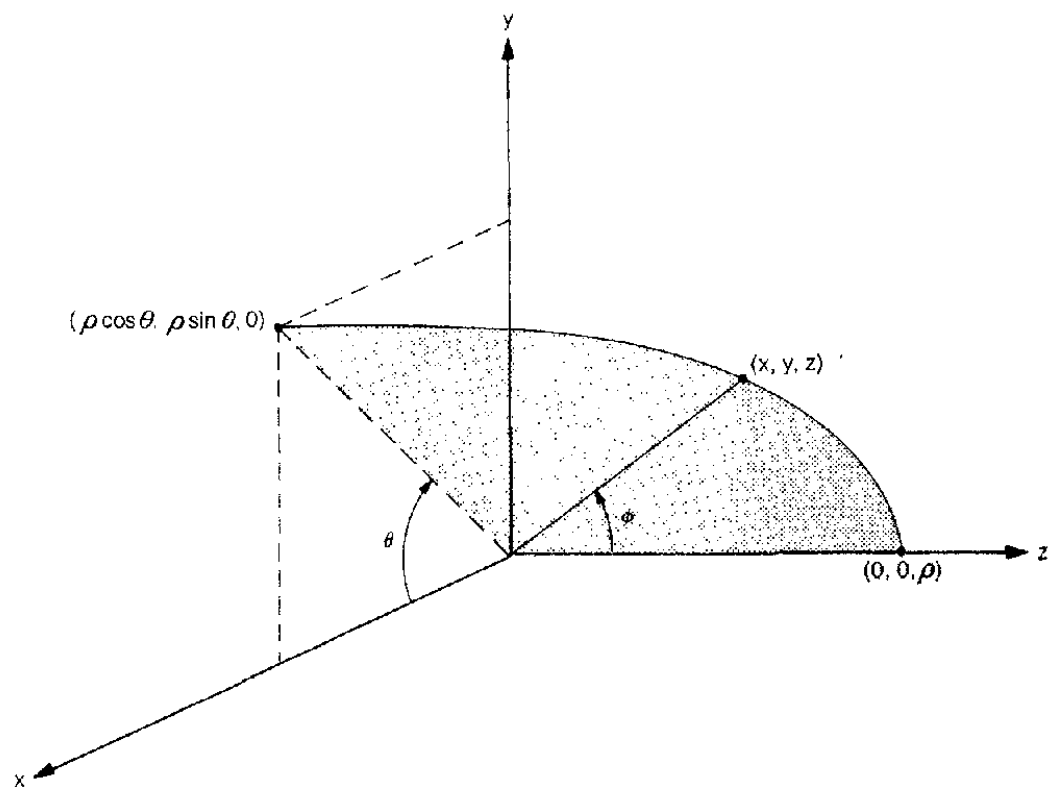

Figure A-2. Spherical coordinates. 
$\left[\alpha_{i j}\right]$ represents a linear transformation of 3 space, $\left[t_{1}, t_{2}, t_{3}\right]$ represents a translation, and $\left[p_{1}, p_{2}, p_{3}\right]$ represents a perspective transformation.

\section{A.2 Direction Cosines}

Direction cosines describe the direction of a vector in 3-space. They are the cosines of the angles between this vector and the three principal coordinate axes. (See Figure A-1.) The direction cosines for the vector $O P$ are $(\cos \alpha, \cos \beta, \cos \gamma)$, where $\alpha$ is the angle between the vector and the positive $\mathrm{x}$-axis, $\beta$ is the angle between the vector and the positive $y$-axis, and $\gamma$ is the angle between the vector and the positive $\mathrm{z}$-axis. If the coordinates of the vector $O P$ in the figure are $(x, y, z)$, then:

$$
\begin{aligned}
& \cos \alpha=\frac{x}{\sqrt{x^{2}+y^{2}+z^{2}}} \\
& \cos \beta=\frac{y}{\sqrt{x^{2}+y^{2}+z^{2}}} \\
& \cos \lambda=\frac{z}{\sqrt{x^{2}+y^{2}+z^{2}}}
\end{aligned}
$$

\section{A.3 Spherical Coordinates}

A point in 3-space can be represented by three Cartesian coordinates $(x, y, z)$ with respect to the standard coordinate axes. It can also be represented by the spherical coordinates $(\rho, \theta, \theta)$, with $\rho \geq 0,0 \leq \theta \leq$ $360^{\circ}$, and $0 \leq \theta \leq 180^{\circ}$.

In Figure A-2, $\theta$ is the angle between the vector $(x, y, z)$ and the positive $z$-axis, $\theta$ is the angle between its orthographic projection on the $x y$ plane and the positive $x$-axis, and $\rho$ is the length of the vector. The equations which relate these two representations are:

$$
\begin{aligned}
& x=\rho \cos \theta \sin \theta \\
& y=\rho \sin \theta \sin \theta \\
& z=\rho \cos \theta
\end{aligned}
$$

\section{A.4 Constraining Equations for Axonometric Projections}

The constraints on an axonometric projection can be viewed as constraints on the direction cosines of the normal of the projection plane. Recall that the direction cosines of this normal are the cosines of the angles between the normal and the principal coordinate axes.

A dimetric projection is defined by the condition that two of the angles between the projection plane and the coordinate axes be equal. This is equivalent to the requirement that the absolute values of two of the direction cosines for the normal be the same, i.e.,

$$
\begin{aligned}
& \frac{|x|}{\sqrt{x^{2}+y^{2}+z^{2}}}=\frac{|y|}{\sqrt{x^{2}+y^{2}+z^{2}}} \text { or } \\
& \frac{|x|}{\sqrt{x^{2}+y^{2}+z^{2}}}=\frac{|z|}{\sqrt{x^{2}+y^{2}+z^{2}}} \text { or } \\
& \frac{|y|}{\sqrt{x^{2}+y^{2}+z^{2}}}=\frac{|z|}{\sqrt{x^{2}+y^{2}+z^{2}}}
\end{aligned}
$$

This implies that the normal of the projection plane for a dimetric projection lies in a plane parallel to one of the six planes $x= \pm y, x= \pm z$, or $y= \pm z$.

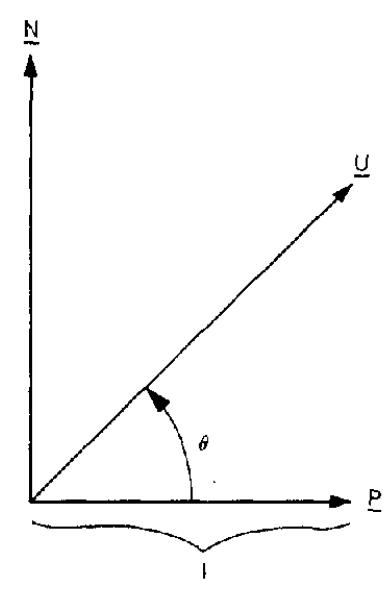

Figure A-3.

An isometric projection is defined by the condition that all three angles between the projection plane and the coordinate axes be equal. This is equivalent to the requirement that the absolute values of the direction cosines for the normal all be identical, i.e.,

$$
\begin{aligned}
\frac{|x|}{\sqrt{x^{2}}+y^{2}+z^{2}} & =\frac{|y|}{\sqrt{x^{2}+y^{2}+z^{2}}} \\
& =\frac{|z|}{\sqrt{x^{2}+y^{2}+z^{2}}}
\end{aligned}
$$

This implies that the normal of the projec- 
tion plane lies along a line parallel to one of the lines $\pm x= \pm y= \pm z$.

\section{A.5 Conditions That Determine an Axonometric Projection}

As discussed in Section 3, the type of an axonometric projection is determined by:

1) the angles between the projection plane and the coordinate axes, or

2) the foreshortening ratios of the three coordinate axes, or

3) the angles between the three coordinate axes.

This section shows that the orientation of the projection plane determines the properties of the projected object, and conversely that either of the properties 2) or 3 ) of the projected object determines the orientation of the projection plane. These relationships are proven by showing that:

a) two coordinate axes intersect the projection plane at the same angle if and only if their foreshortening ratios are the same, and

b) two coordinate axes intersect the projection plane at the same angle if and only if their projections intersect the projection of the third coordinate axis at the same angle. (This assumes that the projection plane is not parallel to one of the principal planes, which would give a degenerate axonometric view, showing only one principal face of the object.)

The first equivalence is easily established with the aid of Figure A-3.

In this diagram, $\boldsymbol{U}$ is a unit vector, $\boldsymbol{P}$ is its projection, $N$ is the unit normal to the projection plane, and $l$ is the foreshortening ratio, that is, the length of $\boldsymbol{P}$. It is clear that $l=\cos \theta$.

If $U_{1}$ and $U_{2}$ are two unit coordinate vectors, then $l_{1}=l_{2}$ if and only if $\theta_{1}=\theta_{2}$; hence a).

To establish the second equivalence, write $\boldsymbol{P}=\boldsymbol{U}-\boldsymbol{N} \sin \Theta$. If $\boldsymbol{U}_{\mathbf{1}}, \boldsymbol{U}_{\mathbf{2}}, \boldsymbol{U}_{3}$ are three principal unit coordinate vectors and $\boldsymbol{P}_{1}, \boldsymbol{P}_{2}, \boldsymbol{P}_{3}$ are their projections, we must show that the angle between $\boldsymbol{P}_{1}$ and $\boldsymbol{P}_{3}$ is equal to the angle between $\boldsymbol{P}_{2}$ and $\boldsymbol{P}_{3}$ if and only if $\theta_{1}=\theta_{2}$. This is equivalent to showing that $\boldsymbol{P}_{1} \cdot \boldsymbol{P}_{3}=\boldsymbol{P}_{2} \cdot \boldsymbol{P}_{3}$ if and only if $\boldsymbol{\theta}_{1}=$ $\Theta_{2}$ (where - denotes the scalar product). But

$$
\begin{aligned}
{ }_{1} P_{3}= & \left(U_{1}-N \sin \Theta_{1}\right) \cdot\left(U_{3}-N \sin \Theta_{3}\right) \\
= & U_{1} \cdot U_{3}-U_{2} \cdot N \sin \Theta_{3} \\
& -U_{3} \cdot N \sin \Theta_{1}+\sin \Theta_{1} \sin \Theta_{3} \\
= & \cos 90^{\circ}-\sin \Theta_{1} \sin \Theta_{3} \\
& -\sin \theta_{3} \sin \theta_{1}+\sin \theta_{1} \sin \Theta_{3} \\
= & -\sin \theta_{1} \sin \Theta_{3}
\end{aligned}
$$

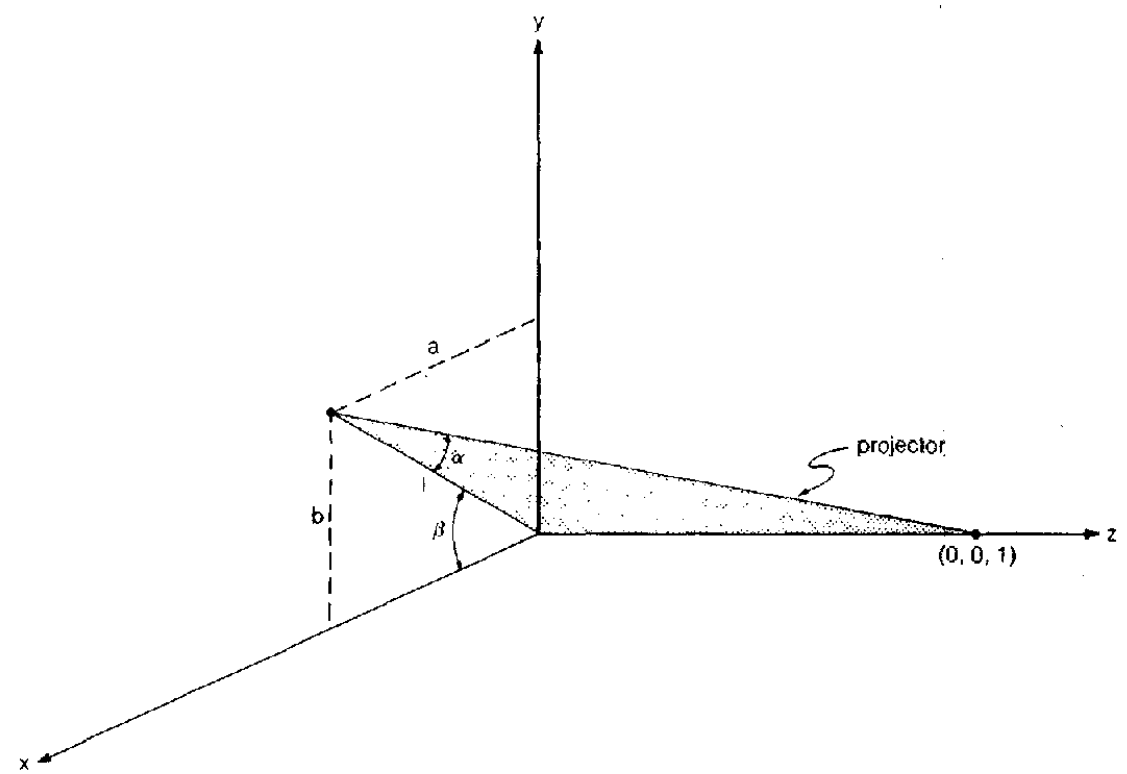

Figure A-4. 
Hence $\cos \theta_{1} \cos \theta_{3} \cos \beta=-\sin \theta_{1} \sin \Theta_{3}$ or $\cos \beta=-\tan \theta_{1} \tan \theta_{3}$, where $\beta$ is the angle between $\boldsymbol{P}_{1}$ and $\boldsymbol{P}_{3}$. Similarly, $\cos \gamma=$ $-\tan \theta_{2} \tan \Theta_{3}$, where $\gamma$ is the angle between $\boldsymbol{P}_{2}$ and $\boldsymbol{P}_{3}$. If $\theta_{1}=\theta_{2}$ then $\cos \beta=\cos \gamma$, and $\beta=\gamma$. Conversely, if $\beta=\gamma$, then $\tan \theta_{1}=$ $\tan \Theta_{2}$, and $\Theta_{1}=\Theta_{2}$; hence $b$ ).

\section{A.6 Conditions That Determine an Oblique Projection}

As discussed in Section 3, an oblique projection is determined by the orientation of the projection plane, the angle between the projectors and the projection plane, and the orientation of the projectors with respect to the projection plane normal. The direction of the projectors can be specified in several ways. This section identifies four sets of parameters that determine the direction of the projectors, and shows their relationships. The parameters are most conveniently specified relative to a coordinate system with two axes in the projection plane and a third along the projection plane normal.

The four sets of parameters are (see Figure A-4):

a) $(\alpha, \beta)$, where $\alpha$ is the angle of intersection of the projector with the projection plane (the xy-plane in Figure $A-4) ; \beta$ is the angle of rotation of the projector about the normal relative to one coordinate axis (the $\mathrm{x}$-axis).

b) $(l, \gamma)$, where $l$ is the length (foreshortening ratio) of the projected unit normal of the projection plane; $\gamma$ is the angle at which this projected normal intersects one of the coordinate axes in the projection plane.

c) $(a, b)$, where $a$ and $b$ are the coordinates in the projection plane of the projected unit normal with respect to the chosen coordinate system. Oblique projection can be thought of as a shear along the projection plane followed by an orthographic projection. In this shear, $a$ and $b$ are the shearing parameters.

d) $(x, y, z)$, where $x, y$, and $z$ are the Cartesian coordinates for a vector. In the Core Graphics System the projectors are specified in this manner.

Equations which relate these pairs of parameters are:
a) and b): $\quad \beta=\gamma, l=\cot \alpha$
a) and c): $\quad a=l \cos \beta, b=l \sin \beta$
a) and d): $\quad(\alpha, \beta)$ determine the projector $(x, y, z)$ relative to a point in the projection plane, since $\left(90^{\circ}+\alpha, \beta\right)$ can be regarded as spherical coordinates for $(x, y, z)$. Hence, $x=\cos \beta \cos \alpha, y=$ $\sin \beta \cos \alpha, z=-\sin \alpha$.

b) and d): $\quad x=l \cos \gamma, y=l \sin \gamma, z=$ -1 . (This vector has the same direction as $(x, y, z)$ in the previous paragraph, but different length.)

\section{A.7 Conditions That Classify Perspective Projections}

As discussed in Section 3, perspective projections can be classified by:

1) the number of the principal coordinate axes that intersect (but do not lie within) the projection plane, or

2) the number of finite principal vanishing points.

This section shows that these two conditions are equivalent.

A vanishing point for a line $L$ is finite if a line through the center of projection, parallel to $L$, pierces the projection plane. Hence, the vanishing point for a coordinate axis is finite if a line through the center of projection parallel to the coordinate axis intersects the projection plane. Clearly this is the case if and only if the coordinate axis intersects the plane; hence 1) and 2) are equivalent.

\section{A.8 Projection Matrices}

The projections discussed in this paper can be expressed in a single $4 \times 4$ matrix. This section describes a simple, straightforward way of deriving this matrix.

The projections have so far been discussed in terms of choosing projection planes as opposed to rotations of the object. As mentioned in the Introduction, this choice was made for consistency with the synthetic camera approach of the Core Graphics System. However, in order to derive a two-dimensional representation of an object, it is simpler to consider an arbitrary projection of an object as a translation and a rotation of this object, followed by a projection onto one of the principal planes. For the discussion below, this principal plane will be the xy-plane. 
A projection of an object can be implemented as a three-step process: 1) a translation, 2) a rotation, and 3) a projection onto a principal plane. Each step can be expressed as a homogenous matrix transformation. Let the translation matrix be $T$, the rotation matrix be $R$, and the projection matrix be $P$. Then, given a point $x$ on the object, the projected point $x_{1}$ is given by $x_{1}$ $=x T \cdot R \cdot P$.

The translation moves the projection plane along its normal so that it intersects the origin. The translation matrix is

$$
T=\left[\begin{array}{cccc}
1 & 0 & 0 & 0 \\
0 & 1 & 0 & 0 \\
0 & 0 & 1 & 0 \\
d \cdot n_{1} & d \cdot n_{2} & d \cdot n_{3} & 1
\end{array}\right]
$$

where $d$ is the shortest distance from the plane to the origin and $\left(n_{1}, n_{2}, n_{3}\right)$ is the projection plane unit normal. Note that $d$ is a signed distance:

$$
\begin{gathered}
d>0 \text { if the normal is directed } \\
\text { towards the origin, } \\
d \leq 0 \text { otherwise. }
\end{gathered}
$$

The rotation is a transformation that turns the translated projection plane into the xy-plane (see Figure A-2).

Assume that $(x, y, z)$ is the (translated) projection plane normal. Then a rotation of $-\theta$ about the $\mathrm{z}$-axis followed by a rotation of $-\theta$ about the $y$-axis will accomplish this rotation ${ }^{15}$. The composite rotation matrix is:

$$
\begin{aligned}
R & =\left[\begin{array}{cccc}
\cos \theta & -\sin \theta & 0 & 0 \\
\sin \theta & \cos \theta & 0 & 0 \\
0 & 0 & 1 & 0 \\
0 & 0 & 0 & 1
\end{array}\right] \\
& {\left[\begin{array}{cccc}
\cos \theta & 0 & \sin \theta & 0 \\
0 & 1 & 0 & 0 \\
-\sin \theta & 0 & \cos \theta & 0 \\
0 & 0 & 0 & 1
\end{array}\right] } \\
& =\left[\begin{array}{cccc}
\cos \theta \cos \theta & -\sin \theta & \cos \theta \sin \theta & 0 \\
\sin \theta \cos \theta & \cos \theta & \sin \theta \sin \theta & 0 \\
-\sin \theta & 0 & \cos \theta & 0 \\
0 & 0 & 0 & 1
\end{array}\right]
\end{aligned}
$$

\footnotetext{
${ }^{15}$ It is common practice to draw one of the principal axes as a vertical line. In order to accomplish this, a third rotation about the $\mathrm{z}$-axis must generally be employed.
}

The projection matrices differ for orthographic, oblique, and perspective projections. These matrices are described below.

An orthographic projection of a point onto the xy-plane is accomplished by the homogeneous matrix

$$
P_{1}=\left[\begin{array}{llll}
1 & 0 & 0 & 0 \\
0 & 1 & 0 & 0 \\
0 & 0 & 0 & 0 \\
0 & 0 & 0 & 1
\end{array}\right]
$$

For orthographic projection, $P=P_{1}$.

An oblique projection of an object onto the xy-plane can be thought of as a shear of the object along the xy-plane, followed by an orthographic projection onto this plane. The shearing operation can be expressed in matrix form:

$$
S=\left[\begin{array}{llll}
1 & 0 & 0 & 0 \\
0 & 1 & 0 & 0 \\
a & b & 1 & 0 \\
0 & 0 & 0 & 1
\end{array}\right]
$$

where $a, b$ are the shearing components discussed in Section A.6. Hence the projection matrix for an oblique projection is $P$ $=S \cdot P_{1}$.

A perspective projection of an object onto the xy-plane can be thought of as a perspective transformation followed by an orthographic projection onto this plane. The matrix of this perspective transformation is:

$$
C=\left[\begin{array}{cccc}
1 & 0 & 0 & 0 \\
0 & 1 & 0 & 0 \\
0 & 0 & 1 & r \\
-c & -d & 0 & 1
\end{array}\right]
$$

where $(c, d,-1 / r)$ is the center of projection in the translated and rotated coordinate system. (The translation by $-c$ and $-d$ positions the center of projection on the $\mathrm{z}$ axis.) The perspective projection matrix is $P=C \cdot P_{1}$. The resulting vector $(x, y, 0, w)$ is finally divided by $w$ to map the result from its $4 \mathrm{D}$ representation to $3 \mathrm{D}$.

\section{A.9 Viewing Specification in the Core Graphics System}

This section briefly describes the viewing functions in the Core Graphics System and gives a few examples of how to use these functions. For additional explanations and 


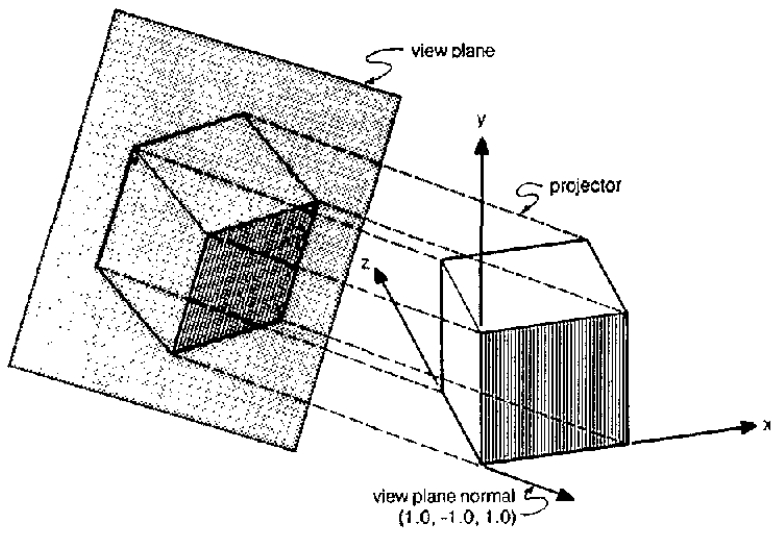

Figure A-5a. Construction of an isometric projection.

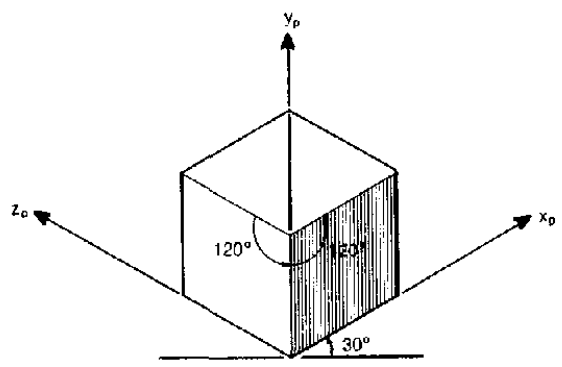

Figure A-5b. Isometric projection resulting from the construction in Figure A-5a. ( $x_{p}, y_{p}$ and $z_{p}$ are the projected coordinate axes.)

programming examples, the reader is referred to BERG78 in this issue.

All the viewing parameters in the Core System that define a projection are specified relative to the view reference point. The view plane normal, (the projection plane normal), is specified as a vector. The view plane is perpendicular to this vector, either through the view reference point or at a distance from it, specified by the view plane distance. The view up vector defines the direction that is "up" in the projection plane and that is to be vertical in the resulting projection.

A parallel projection is specified by a vector that indicates the direction of its projectors. A perspective projection is specified by its center of projection, which is defined relative to the view reference point.

\section{Example 1:}

The following code will generate an isometric view of a cube. The cube, along with the

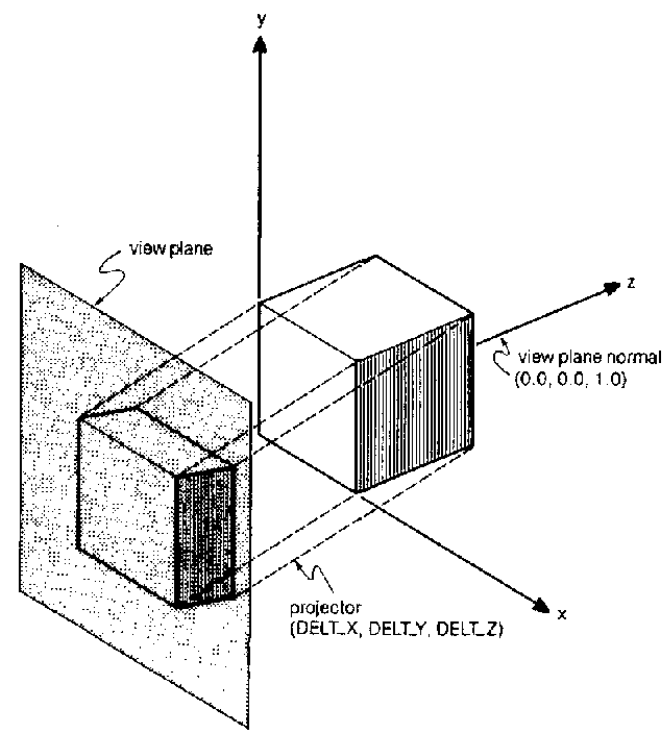

Figure A-6a. Construction of an oblique projection.

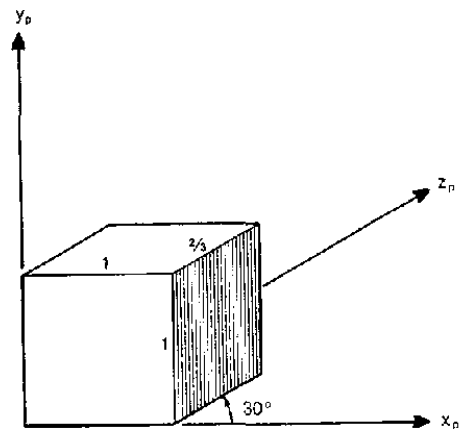

Figure A-6b. Oblique projection resulting from the construction in Figure A-6a. $\left(x_{p}, y_{p}\right.$ and $z_{p}$ are the projected coordinate axes.)

view plane, the view plane normal, the projectors, and the resulting projected cube is illustrated in Figure A-5.

(* The viewing parameters will be specified relative to the origin. ${ }^{*}$ )

VIEW_REFERENCE_POINT $(0.0$, $0.0,0.0$ );

(* An isometric view is obtained if the absolute values of the components of the view plane normal are all equal. ${ }^{*}$ ) VIEW_PLANE_NORMAL $(1.0,-1.0,1.0)$;

(* Although the distance of the view plane to the object does not affect the resulting projection, the view plane is positioned at a distance 
from the origin to correspond to Figure A-5a. *)

VIEW_PLANE_DISTANCE(-2.5);

(* The $y$-axis should be vertical in the resulting projection. *)

VIEW_UP_3 $3(0.0,1.0,0.0)$

(* The projectors are parallel to the view plane normal. *)

PARALLEL(1.0, -1.0, 1.0);

(* The routine CUBE generates the cube *) CUBE;

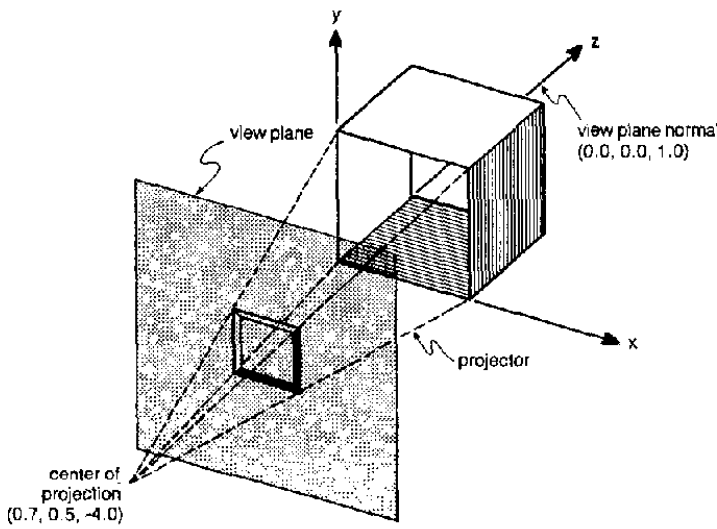

Figure A-7a. Construction of a one-point perspective projection.

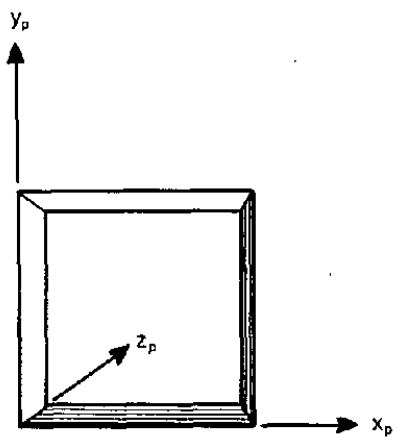

Figure A-7b. One-point perspective projection resulting from the construction in Figure A-7a. $\left(x_{p}, y_{p}\right.$ and $\mathrm{z}_{\mathrm{p}}$ are the projected coordinate axes.)

\section{Example 2:}

The following code will generate an oblique view of a cube. The cube, along with the view plane, the view plane normal, the projectors, and the resulting projected cube is illustrated in Figure A-6.

VIEW_REFERENCE_POINT $(0.0$, $0.0,0.0)$;
(* The side of the cube parallel to the xy-plane is projected to true size. $\left.{ }^{*}\right)$

VIEW _PLANE_NORMAL $(0.0,0.0,1.0)$;

(* Although the distance of the view plane to the object does not affect the resulting projection, the view plane is positioned at a distance from the origin to correspond to Figure A-6a. *)

VIEW_PLANE_DISTANCE(-2.0);

(* The y-axis should be vertical in the resulting projection. $\left.{ }^{*}\right)$

VIEW_UP_3(0.0,1.0,0.0);

(* The foreshortening ratio of the receding axis is $2 / 3$ and the axis is projected at a $30^{\circ}$ angle with the projected $x$-axis. ${ }^{*}$ )

DELT $X:=\operatorname{COS}(30.0) * 2.0 / 3.0$;

DELT_Y: $=\operatorname{SIN}(30.0) * 2.0 / 3.0$;

DELT_Z $Z:=-1.0$

PARALLEL(DELT_X, DELT_Y,

DELT _.Z);

CUBE;

\section{Example 3:}

The following code will generate a onepoint perspective view of a cube. The cube, along with the view plane, the view plane normal, the projectors, and the resulting projected cube is illustrated in Figure A-7.

VIEW_REFERENCE_POINT $(0.0,0.0$, $0.0)$;

(* The view plane is parallel to the $x y-$ plane. ${ }^{*}$ )

VIEW_PLANE_NORMAL $(0.0,0.0,1.0)$;

VIEW_PLANE_DISTANCE $(-2.0)$;

${ }^{*}$ The $y$-axis should be vertical in the resulting projection. *)

VIEW_UP_3 $3(0.0,1.0,0.0)$;

(* The center of projection is displaced slightly horizontally with respect to the center of the cube. *)

PERSPECTIVE $(0.7,0.5,-4.0)$; CUBE;

\section{REFERENCES}

Anth22 Anthony, G.C. An introduction to the graphic language, D.C. Heath \& Co., New York, 1922, 166pp.

Berg78 Bergeron, R.D., BoNo, P.R., AND Foley, J.D. "Graphics programming using the Core System," Comput. Surv. 10, 4 (Dec., 1978), 389-443.

Chin75 Ching, F. Architectural graphics, Van Nostrand Reinhold Co., New York, 1975, 128pp.

Chur64 ChurCH, A.E. Elements of descriptive geometry, A.S. Barnes \& Co., New York, $1864,192 \mathrm{pp}$.

Chur11 Church, A.E., and Bartlett, G.M. Elements of descriptive geometry, American Book Co., New York, 1911, 286pp.

Cool40 CoOLIDGE, J.L. A history of geometrical 
methods, Clarendon Press, Oxford, 1940, 451pp.

Coul66 Coulin, c. Step-by-step perspective drawing, Van Nostrand Reinhold Co., New York, 1966, 112pp.

Croz21 CRozET, C. Descriptive geometry, A.T. Goodrich Co., New York, 1821.

Cutr23 CutTer, L,E. Descriptive geometry, McGraw-Hill, Inc., New York, 1923, 244pp.

Dame64 D'Amelo, J. Perspective drawing handbook, Leon Amiel, New York, 1964, $96 \mathrm{pp}$.

Davi26 Davies, C. Elements of descriptive geometry, Wiley \& Long, New York, 1826 , $174 \mathrm{pp}$.

Düre25 DürER, A. Unterweisung der Messung mit dem Zyrkel und Rychtscheyd, StockerSchmid, Zuirich, 1525, 217pp.

Eves63 EVEs, H. A survey of geometry, Vol. 1, Allyn \& Bacon, Inc., Boston, 1963, 489pp.

Eves65 Eves, H. A survey of geometry, Vol. 2, Allyn \& Bacon, Inc., Boston, 1965, 496pp.

Eves69 Eves, H. An introduction to the history of mathematics, Holt, Rinehard \& Winston, New York, 1969, 464pp.

Fran54 FrankFort, $\mathrm{H}$. The art and architecture of the ancient Orient, Penguin Books, Inc., New York, 1954, 279pp.

Fren47 FrENCH, T.E. Engineering drawing, Rev. by C.J. Vierck, McGraw-Hill, Inc., New York, 1947, 694pp.

FREN70 FrENCH, T.E., AND Vierck, C.J. Graphic science and design, McGraw-Hill Inc., New York, 1970, 788pp.

Giac61 Giachino, J.W., and Beukema, H.J. Drafting and graphics, American Technical Society, Chicago, 1961, 778pp.

Gies58 Giesecke, F.E., Mitchell, A., AND SPENCER, H.C. Technical drawing, Macmillan Inc., New York, 1958, 849pp.

Grl.74 GILl, R.W. Basic perspective, Thames and Hudson, London, $1974,96 \mathrm{pp}$.

GSPC77 GSPC "Status report of the Graphics Standards Planning Committee of ACM/ SIGGRAPH," Comput. Gr. 11, 3 (1977), II-1 - II-177.

Hamm71 Hammond, R.H., Buck, C.P., Rogers, W.B., WALSH, G.W., JR., AND ACKERT, H.P. Engineering graphics, Ronald Press Co., New York, 1971, 648pp.

HoEl63 HoELSChER, R.P., SPRINGER, C.H., AND DoBRovolnY, J. Basic drawing for engineering technology, John Wiley \& Sons, Inc., New York, 1963, 396pp.

IviN75 IVINS, W.M., JR. On the rationalization of sight, Da Capo Press, Inc., New York, $1975,160 \mathrm{pp}$.

JANS62 JANSON, H.W. History of art, Harry N. Abrams, Inc., New York, 1962, 572pp.

Jord29 Jordan, H.H., and PORTER F.M. De. scriptive geometry, Grim and Co., Boston, $1929,349 \mathrm{pp}$.

JORD35 JORDAN, H.H., AND HOELSCHER, R.P.
Engineering drawing, John Wiley \& Sons, Inc., New York, $1935,528 \mathrm{pp}$.

KeLl49 Kellaway, G.P. Map projections, E.P. Dutton \& Co., Inc., New York, 1949, 127pp.

Kurn54 KuINe, M. Mathematics in western culture, George Allen and Unwin, Ltd., London, 1954 , 484pp.

Lanc70 Lanczos, C. Space through the ages, Academic Press, London, 1970, 320pp.

Leve62 LeVENS, A.S. Graphics, John Wiley \& Sons, Inc., New York, 1962, 743pp.

LUZA57 LUZADDER, W.J. Graphics for engineers, Prentice-Hall, Inc., Englewood Cliffs, N.J., $1957,597 \mathrm{pp}$.

MaRT68 MarTin, C.L. Design graphics, Macmillan Inc., New York, 1968, 307pp.

Mild41 Miller, H.W. Descriptive geometry, John Wiley \& Sons, Inc., New York, 1941, 254pp.

NewM56 NEWMAN, J.R. (ED.) The world of mathematics, Simon and Schuster, New York, 1956.

Newm73 Newman, W.M., and Sproull, R.F. Principles in interactive computer graphics, McGraw-Hill, Inc., New York, 1973, $607 \mathrm{pp}$.

Newm78 Newman, W.M., and VaN Dam, A. "Recent efforts towards graphics standardization", Comput. Surv. 10, 4 (Dec. 1978), 365-380.

OLes78 OLEs, P.S. Architectural illustration, Van Nostrand Reinhold Co., New York, 1978.

Parr60 Parrot, A. Sumer, Gallimard, Paris, 1960, 392pp.

Rich72 RichardUs, P., AND Adter, R.K. Map projections, Elsevier North-Holland Inc., New York, 1972, 174pp.

Risi77 Rising, J.S., ALMFELdT, M.W., AND DEJoNG, P.S. Engineering graphics: communication, analysis, and creative design, Kendall-Hunt, Dubuque, Iowa, 1977.

ROGE76 ROGERS, D.F., AND ADAMS, J.A. Mathematical elements for computer graphics, McGraw-Hill, Inc., New York, $1976,239 \mathrm{pp}$.

Rule61 RULe, J.T., AND Coons, S.A. Graphics, McGraw-Hill, Inc., New York, 1961, 484pp.

Smit36 SMITH, W.G. Practical descriptive geometry, McGraw-Hill Inc., New York, 1936, $275 \mathrm{pp}$.

Stee48 SteERS, J.A., And Debenham, F. Study of map projections, University of London Press, Ltd., London, 1948, 292pp.

WaTT46 WATTS, E.F., AND RULE, J.T. Descriptive geometry, Prentice-Hall, Inc., Englewood Cliffs, N.J., 1946, 301pp.

WhIt67 WhITE, J. The birth and rebirth of pictorial space, Faber and Faber, London, $1967,289 \mathrm{pp}$.

WILl98 WILLSON, F.N. Theoretical and practical graphs, Macmillan, Inc., New York, 1898, 293pp. 